\title{
Why many theories of shock waves are necessary. Convergence error in formally path-consistent schemes
}

\author{
Manuel J. Castro \\ Departamento de Análisis Matemático, Universidad de Málaga, 29071 Málaga, Spain. \\ Castro@anamat.cie.uma.es \\ Philippe G. LeFloch \\ Laboratoire J.-L. Lions \& Centre National de la Recherche Scientifique, \\ Université de Paris 6, 4 Place Jussieu, 75252 Paris, France. LeFloch@ann.jussieu.fr \\ María Luz Muñoz-Ruiz \\ Departamento de Matemática Aplicada, Universidad de Málaga, 29071 Málaga, Spain. \\ Munoz@anamat.cie.uma.es \\ Carlos Parés \\ Departamento de Análisis Matemático, Universidad de Málaga, 29071 Málaga, Spain. \\ Pares@anamat.cie.uma.es
}

\begin{abstract}
We are interested in nonlinear hyperbolic systems in nonconservative form arising in fluid dynamics, and, for solutions containing shock waves, we investigate the convergence of finite difference schemes applied to such systems. According to Dal Maso, LeFloch, and Murat's theory, a shock wave theory for a given nonconservative system requires prescribing a priori a family of paths in the phase space. In the present paper, we consider schemes that are formally consistent with a given family of paths, and we investigate their limiting behavior as the mesh is refined. we first generalize to systems a property established earlier by Hou and LeFloch for scalar conservation laws, and we prove that nonconservative schemes generate, at the level of the limiting hyperbolic system, an convergence error source-term which, provided the total variation of the approximations remains uniformly bounded, is a locally bounded measure. This convergence error measure is supported on the shock trajectories and, as we demonstrate here, is usually "small". In the special case that the scheme converges in the sense of graphs - a rather strong convergence property often violated in practice - then this measure source-term vanishes. We also discuss the role of the equivalent equation associated with a difference scheme; here, the distinction between scalar equations and systems appears most clearly since, for systems, the equivalent equation of a scheme that is formally path-consistent depends upon the prescribed family of paths. The core of this paper is devoted to investigate numerically the approximation of several (simplified or full) hyperbolic models arising in fluid dynamics. This leads us to the
\end{abstract}

Published in: J. Comput. Phys. 227 (2008), 8107-8129. 
conclusion that for systems having nonconservative products associated with linearly degenerate characteristic fields, the convergence error vanishes. For more general models, this measure is evaluated very accurately, especially by plotting the shock curves associated with each scheme under consideration; as we demonstrate, plotting the shock curves provide a convenient approach for evaluating the range of validity of a given scheme.

Key words: nonconservative hyperbolic system, shock wave, family of paths, equivalent equation, convergence error measure, formally path-consistent scheme. 


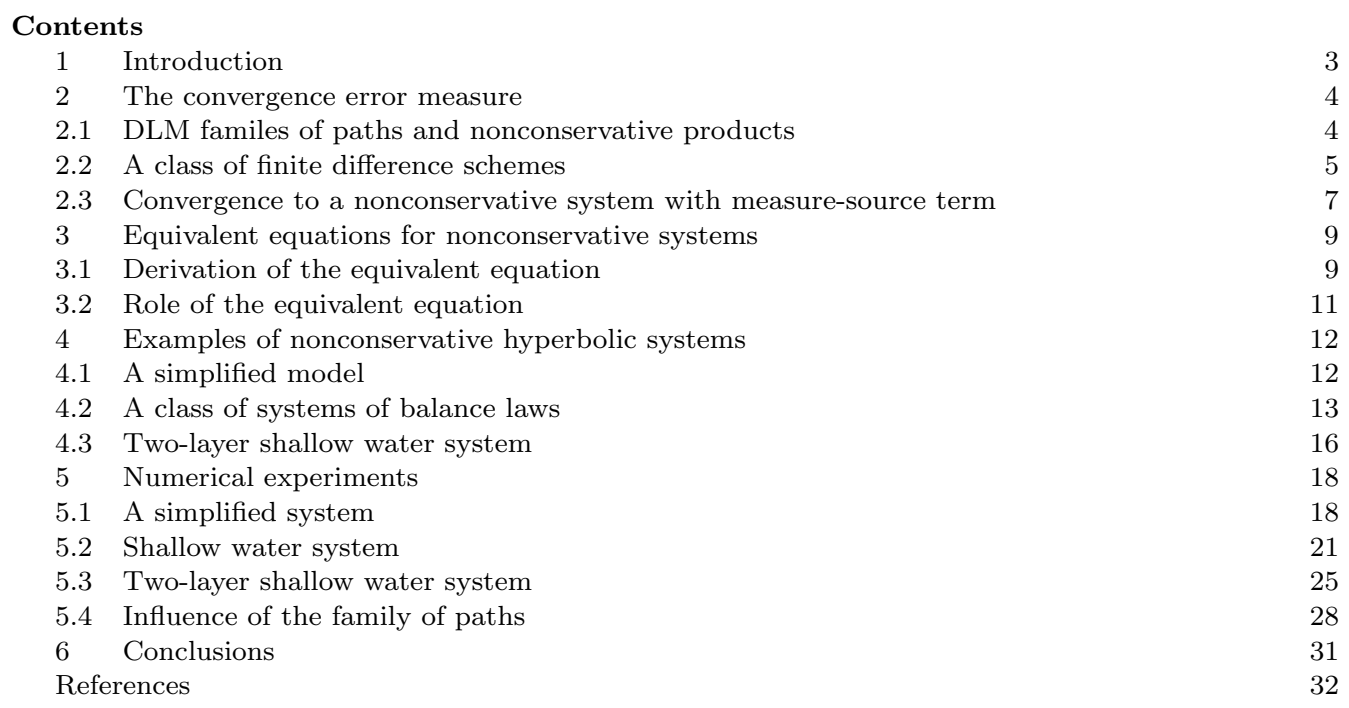

\section{Introduction}

A number of non-conservative hyperbolic models have been introduced in fluid dynamics to serve as (simplified) models of two-phase or two-layer flows. Our objective in the present paper is to address the fundamental question whether finite difference schemes for nonconservative systems converge toward correct weak solutions containing shock waves. Addressing this important issue requires detailed numerical computations which we carry out here. The nonconservative hyperbolic systems under consideration have the general form

$$
u_{t}+A(u) u_{x}=0, \quad u=u(t, x) \in \mathbb{R}^{N},
$$

where $u$ is the vector-unknown and $A=A(u)$ is a smooth, $N \times N$ matrix-valued map $A$ which admits real eigenvalues $\lambda_{1}<\ldots<\lambda_{N}$ and a basis of eigenvectors $r_{1}, \ldots, r_{N}$. We are interested in solving the initial value problem associated with some initial condition

$$
u(0, x)=u_{0}(x), \quad x \in \mathbb{R} .
$$

The solutions of nonlinear hyperbolic systems are generally discontinuous; due to the non-divergence form of the equations the notion of solutions in the sense of distributions can not be used, and weak solutions satisfying (1.1) are defined in the sense introduced by LeFloch $[18,19,20,21,23]$ and Dal Maso, LeFloch, and Murat [13] (cf. Section 2 below for a brief review of the theory).

Generally speaking, solutions to (1.1) depend upon regularization mechanisms; for instance, different approximation schemes may converge toward different solutions, and for this reason in developing the well-posedness theory, higher-order regularization effects such as viscosity, capillarity, relaxation terms, must be taken into account in the modeling. For instance, for continuous models one may consider the regularization 


$$
u_{t}^{\epsilon}+A\left(u^{\epsilon}\right) u_{x}^{\epsilon}=R^{\epsilon},
$$

where $R^{\epsilon}$ depends upon higher-derivatives of $u$ together with (one or several) small-scale parameter(s) $\epsilon$; the physically meaningful solutions are defined as the singular limits

$$
u:=\lim _{\epsilon \rightarrow 0} u^{\epsilon} .
$$

Furthermore, as established in [19], shock waves in such solutions are determined by traveling wave solutions to (1.3), that is, Rankine-Hugoniot relations for shock waves are determined from the given regularization.

In the present paper, we demonstrate that while, for certain simplified models, solutions are actually stable upon regularization and the detailed knowledge of the right-hand side of (1.3) is unnecessary, however for general systems such as the "full" systems of two-phase flows, the general DLM theory is necessary. Still, as pointed out by Hou and LeFloch [16] — who focused attention on the same issues for nonconservative formulations of scalar hyperbolic equations - and by Hayes and LeFloch [14,15] and LeFloch and Mohammadian [25] — who studied the effect of diffusive and dispersive terms - the effects of the regularization $R^{\epsilon}$ may be difficult to pinpoint in practice. In view of the fact that the models under study are derived from modeling approximation assumptions, this fully justifies the use of a numerical strategy based on a direct discretization of the nonconservative hyperbolic models (1.1). Our conclusions, therefore, justify to search for robust and efficient high-order schemes for the approximation of nonconservative systems. In particular, Berthon and Coquel [1,2] and Chalons and Coquel [11], have introduced various numerical strategies for models of complex fluid flows including turbulence models, while Parés [30] and Muñoz-Ruiz and Parés [28] have developed many important applications.

Finally, for anther standpoint to the theory and the numerical analysis of nonconservative products, we refer to Berthon, Coquel, and LeFloch [3] who connected the theory of nonconservative products with the concept of a kinetic relation [22]. They introduced a general framework to handle nonconservative systems; this framework encompasses a large number of examples arising in the applications. In particular, they rigorously analyzed a typical model of turbulent fluid dynamics by establishing the existence and properties of a physically relevant family of traveling waves and deriving the corresponding kinetic function.

\section{The convergence error measure}

\subsection{DLM familes of paths and nonconservative products}

Let $\Omega$ be an open subset of $\mathbb{R}^{N}$ and $g: \Omega \rightarrow \Omega$ be a smooth mapping. Given a function with bounded variation $u: \mathbb{R} \rightarrow \Omega$, the definition introduced by Dal Maso, LeFloch, and Murat [13] allows one to define products of the form $g(u) \frac{d u}{d x}$ provided a family of Lipschitz continuous paths $\Phi:[0,1] \times \Omega \times \Omega$ is prescribed, which must satisfy certain natural regularity conditions, in particular

$$
\begin{gathered}
\Phi\left(0 ; u_{l}, u_{r}\right)=u_{l}, \quad \Phi\left(1 ; u_{l}, u_{r}\right)=u_{r} \\
\Phi\left(s ; u_{l}, u_{l}\right)=u_{l}
\end{gathered}
$$


for all $s \in[0,1]$ and $u_{l}, u_{r} \in \Omega$. The nonconservative product, denoted by $\left[g(u) \frac{d u}{d x}\right]_{\Phi}$, is defined as a bounded measure, which is absolutely continuous with respect to the total variation measure of the function $u$ and, in particular, coincides with the distributional derivative $\frac{d}{d x} f$ in the special case of a conservative product, for which $g(u)=D f(u)$ for some $f$.

The DLM theory was applied to nonconservative systems of the form (1.1); the Riemann problem was solved and, later, the general Cauchy problem [24]. In the course of this analysis, the notion of $\Phi$-completion $\left(X, U_{\Phi}\right)$ of the graph of a BV function $u$ was introduced. The key stability result in [13] is the following: if $u^{\Delta}: \mathbb{R} \rightarrow \Omega$ is a sequence of BV functions with uniformly bounded amplitude and total variation

$$
\sup _{\mathbb{R}}\left|u^{\Delta}\right|+T V_{\mathbb{R}}\left(u^{\Delta}\right) \lesssim 1,
$$

converging almost everywhere to a limit function $u: \mathbb{R} \rightarrow \Omega$, then a sufficient condition for the corresponding nonconservative products to converge

$$
\left[g\left(u^{\Delta}\right) \frac{d u^{\Delta}}{d x}\right]_{\Phi} \rightarrow\left[g(u) \frac{d u}{d x}\right]_{\Phi}
$$

in the weak-star sense of measures, is that their $\Phi$-completions $\left(X^{\Delta}, U_{\Phi}^{\Delta}\right)$ converge in the uniform distance of graphs precisely to the $\Phi$-completion $\left(X, U_{\Phi}\right)$ of the limit $u$.

\subsection{A class of finite difference schemes}

For the approximation of nonconservative systems we introduce here a general family of numerical schemes which includes, in particular, three classes of schemes of particular interest: Godunov, Roe, and Lax-Friedrichs.

For the discretization of the initial value problem (1.1)-(1.2), we introduce computing cells $I_{i}=\left[x_{i-1 / 2}, x_{i+1 / 2}\right]$ and, for simplicity in the presentation, we assume that these cells have constant size $\Delta=\Delta x$. We also define $x_{i+1 / 2}=i \Delta x$ and $x_{i}=(i-1 / 2) \Delta x$, the latter being the center of the cell $I_{i}$. Finally we denote by $\Delta t$ the (constant) time length and we set $t^{n}=n \Delta t$. We denote by $u_{i}^{n}$ the approximation of the cell averages of the exact solution provided by the numerical scheme:

$$
u_{i}^{n} \cong \frac{1}{\Delta x} \int_{x_{i-1 / 2}}^{x_{i+1 / 2}} u\left(t^{n}, x\right) d x .
$$

We are interested in schemes of the general form

$$
u_{i}^{n+1}=u_{i}^{n}-\frac{\Delta t}{\Delta x}\left(M_{i-1 / 2}^{n,+}+M_{i+1 / 2}^{n,-}\right),
$$

where

$$
M_{i+1 / 2}^{n, \pm}=M^{ \pm}\left(u_{i-q}^{n}, \ldots, u_{i+p}^{n}\right) .
$$

From now on a DLM family of paths $\Phi$ for the nonconservative system (1.1) is fixed. Following Parés [30], we consider formally path-consistent schemes, i.e. schemes that are consistent with the family of paths $\Phi$ in the following sense: $M^{-}$and $M^{+}$are Lipschitz continuous mappings from $\Omega^{p+q+1}$ to $\Omega$ satisfying:

$$
M^{ \pm}(u, \ldots, u)=0, \quad u \in \Omega,
$$


and for every $u_{i} \in \Omega, i=-q, \ldots, p$,

$$
M^{-}\left(u_{-q}, \ldots, u_{p}\right)+M^{+}\left(u_{-q}, \ldots, u_{p}\right)=\int_{0}^{1} A\left(\Phi\left(s ; u_{0}, u_{1}\right)\right) \frac{\partial \Phi}{\partial s}\left(s ; u_{0}, u_{1}\right) d s .
$$

These conditions provide a generalization to the concept of conservative scheme introduced by Lax for systems of conservation laws and, for this reason, were originally called "path-conservative" in [30]. As we will see, this definition -although quite natural- needs to be handled carefully.

It is convenient to assume some particular structure on the given family of paths. Precisely, we assume that the matrix $A$ and the paths $\Phi$ satisfy the following restrictions:

(R1) Given an integral curve $\gamma$ of a linearly degenerate field and $u_{l}, u_{r} \in \gamma$, the path $\Phi\left(s ; u_{l}, u_{r}\right)$ is a parametrization of the arc of $\gamma$ connecting $u_{l}$ and $u_{r}$.

(R2) Given an integral curve $\gamma$ of a genuinely nonlinear field and $u_{l}, u_{r} \in \gamma$, with $\lambda\left(u_{l}\right)<$ $\lambda\left(u_{r}\right)$, being $\lambda(u)$ the corresponding eigenvalue, the path $\Phi\left(s ; u_{l}, u_{r}\right)$ is a parametrization of the arc of $\gamma$ connecting $u_{l}$ and $u_{r}$.

(R3) Let us denote by $\mathcal{R P} \subset \Omega \times \Omega$ the set of pairs $\left(u_{l}, u_{r}\right)$ for which the Riemann problem

$$
\left\{\begin{array}{l}
u_{t}+A(u) u_{x}=0, \\
u(x, 0)= \begin{cases}u_{l}, & x<0, \\
u_{r}, & x>0,\end{cases}
\end{array}\right.
$$

has a unique self-similar weak solution composed by $N$ (possibly trivial) simple waves connecting $N+1$ intermediate constant states

$$
u_{0}=u_{l}, u_{1}, \ldots, u_{N-1}, u_{N}=u_{r} .
$$

Given $\left(u_{l}, u_{r}\right) \in \mathcal{R P}$, the curve described by the path $\Phi\left(\cdot ; u_{l}, u_{r}\right)$ is equal to the union of those corresponding to the paths $\Phi\left(\cdot ; u_{j-1}, u_{j}\right), j=1, \ldots, N$.

We now present several schemes of particular interest in the present paper. First of all, the Godunov method for the nonconservative system (1.1) takes the form (2.5) with

$$
\begin{aligned}
& M_{i+1 / 2}^{n,-}=\int_{0}^{1} A\left(\Phi\left(s ; u_{i}^{n}, u_{i+1 / 2}^{n}\right)\right) \frac{\partial \Phi}{\partial s}\left(s ; u_{i}^{n}, u_{i+1 / 2}^{n}\right) d s, \\
& M_{i+1 / 2}^{n,+}=\int_{0}^{1} A\left(\Phi\left(s, u_{i+1 / 2}^{n}, u_{i+1}^{n}\right)\right) \frac{\partial \Phi}{\partial s}\left(s ; u_{i+1 / 2}^{n}, u_{i+1}^{n}\right) d s,
\end{aligned}
$$

where $u_{i+1 / 2}^{n}$ is the (constant) value at $x=0$ of the solution of the Riemann problem consisting of (1.1) with initial condition:

$$
u(x, 0)= \begin{cases}u_{i}^{n}, & x<0, \\ u_{i+1}^{n}, & x>0 .\end{cases}
$$

In the derivation it is important to assume a "CFL-1/2 condition", as noted in [28].

In the case in which the solution of the Riemann problem is discontinuous at $x=0$, such discontinuity has to be stationary and we can replace $u_{i+1 / 2}^{n}$ either by the limit of the solution to the left or to the right of 0. 
Secondly, Roe methods provide linear approximate Riemann solvers. In view of the framework in [13] the following generalization to Roe's standard approach was proposed by LeFloch [21]. Given a family of paths $\Phi$, a function $A_{\Phi}: \Omega \times \Omega \mapsto \mathcal{M}_{N \times N}(\mathbb{R})$ is called a (generalized) Roe linearization if for all $u_{l}, u_{r} \in \Omega$ the following properties hold:

(i) $A_{\Phi}\left(u_{l}, u_{r}\right)$ has $N$ distinct real eigenvalues,

(ii) $A_{\Phi}\left(u_{l}, u_{l}\right)=A\left(u_{l}\right)$,

(iii) $A_{\Phi}\left(u_{l}, u_{r}\right) \cdot\left(u_{r}-u_{l}\right)=\int_{0}^{1} A\left(\Phi\left(s ; u_{l}, u_{r}\right)\right) \frac{\partial \Phi}{\partial s}\left(s ; u_{l}, u_{r}\right) d s$.

Once a Roe linearization has been chosen, the corresponding Roe scheme takes the form (2.5) with

$$
\begin{aligned}
& M_{i+1 / 2}^{n,-}=A_{i+1 / 2}^{n,-} \cdot\left(u_{i+1}^{n}-u_{i}^{n}\right), \\
& M_{i+1 / 2}^{n,+}=A_{i+1 / 2}^{n,+} \cdot\left(u_{i+1}^{n}-u_{i}^{n}\right),
\end{aligned}
$$

where $A_{i+1 / 2}^{n}=A_{\Phi}\left(u_{i}^{n}, u_{i+1}^{n}\right)$,

$$
\mathcal{L}_{i+1 / 2}^{n, \pm}=\left[\begin{array}{ccc}
\left(\lambda_{i+1 / 2,1}^{n}\right)^{ \pm} & & 0 \\
& \ddots & \\
0 & & \left(\lambda_{i+1 / 2, N}^{n}\right)^{ \pm}
\end{array}\right]
$$

and

$$
A_{i+1 / 2}^{n, \pm}=\mathcal{K}_{i+1 / 2}^{n} \mathcal{L}_{i+1 / 2}^{n, \pm}\left(\mathcal{K}_{i+1 / 2}^{n}\right)^{-1}
$$

Here, $\mathcal{L}_{i+1 / 2}^{n}$ denotes the diagonal matrix whose coefficients are the eigenvalues of $A_{i+1 / 2}^{n}$, $\lambda_{i+1 / 2,1}^{n}<\lambda_{i+1 / 2,2}^{n}<\cdots<\lambda_{i+1 / 2, N}^{n}$, and $\mathcal{K}_{i+1 / 2}^{n}$ a $N \times N$ matrix whose columns are associated eigenvectors.

Third, a generalization of the classical Lax-Friedrichs method to (1.1) is given by (2.5) with the choice:

$$
\begin{aligned}
& M_{i+1 / 2}^{n,-}=\int_{0}^{1} \widehat{A}^{-}\left(\Phi\left(s ; u_{i}^{n}, u_{i+1}^{n}\right)\right) \frac{\partial \Phi}{\partial s}\left(s ; u_{i}^{n}, u_{i+1}^{n}\right) d s \\
& M_{i+1 / 2}^{n,+}=\int_{0}^{1} \widehat{A}^{+}\left(\Phi\left(s, u_{i}^{n}, u_{i+1}^{n}\right)\right) \frac{\partial \Phi}{\partial s}\left(s ; u_{i}^{n}, u_{i+1}^{n}\right) d s
\end{aligned}
$$

where

$$
\widehat{A}^{-}(u)=\frac{1}{2}\left(-\frac{\Delta x}{\Delta t} I d+A(u)\right), \quad \widehat{A}^{+}(u)=\frac{1}{2}\left(\frac{\Delta x}{\Delta t} I d+A(u)\right),
$$

being $I d$ the $N \times N$ identity matrix.

\subsection{Convergence to a nonconservative system with measure-source term}

We denote by $u^{\Delta}$ the sequence of piecewise constant approximate solutions generated by a finite difference scheme of the form described above. By extending the arguments in Hou and LeFloch [16] we can prove:

Claim 1 Consider a nonconservative hyperbolic system (1.1) together with a given family of paths $\Phi$. Suppose that $u^{\Delta}$ is a sequence of approximate solutions constructed by 
one of the finite difference schemes described in Subsection 2.2 and satisfying the bounds (2.3) uniformly in time. Suppose that the scheme is formally consistent with the family of paths $\Phi$. Then, given any subsequence of $u^{\Delta}$ converging almost everywhere to some limit, denoted by $v$, the following holds:

(i) There exists a bounded measure $\mu_{v}: \mathbb{R}_{+} \times \mathbb{R} \rightarrow \mathbb{R}^{N}$ (called the convergence error measure) such that the limit $v$ satisfies the following hyperbolic system with sourceterm

$$
v_{t}+\left[A(v) v_{x}\right]_{\Phi}=\mu_{v}
$$

(ii) Moreover, when the $\Phi$-completion of the graphs of $u^{\Delta}$ converges in the uniform sense of graphs towards the $\Phi$-completion of the limit $v$, i.e.

$$
\left(X^{\Delta}, U_{\Phi}^{\Delta}\right) \rightarrow\left(Y, V_{\Phi}\right)
$$

then the convergence error measure $\mu_{v}$ vanishes identically and $v$ is a weak solution to the system

$$
v_{t}+\left[A(v) v_{x}\right]_{\Phi}=0
$$

The above result can be interpreted as a "nonconservative extension" to the classical Lax-Wendroff theorem for systems of conservation laws [17]. It should be observed that the convergence in the sense of graphs is very strong; it does hold for the Glimm and front tracking schemes, but usually fails for finite difference schemes.

Our main objective in the present paper is to investigate the source and the amplitude of this convergence error, which can be measured in terms of the measure $\mu_{v}$ or, equivalently, in terms of the Rankine-Hugoniot curves associated with the given scheme. Indeed, computing numerically the shock curves associated with various schemes of interest is one of the main purposes of this work.

Proof. We follow Hou and LeFloch [16] and decompose the scheme into a part that converges to the hyperbolic system and an error term, and we then rely on stability results established in the general DLM theory [13]. We need to prove that

$$
\int_{0}^{\infty} \int_{-\infty}^{\infty} v(t, x) \varphi_{t}(t, x) d x d t-\int_{0}^{\infty}\left\langle\left[A(v(t, \cdot)) v_{x}(t, \cdot)\right]_{\Phi}, \varphi(t, \cdot)\right\rangle d t=0
$$

for all compactly supported test-function $\varphi=\varphi(t, x)$.

We set

$$
\varphi_{i}^{n}=\varphi\left(t^{n}, x_{i}\right), \quad \varphi_{i+1 / 2}^{n}=\varphi\left(t^{n}, x_{i+1 / 2}\right)
$$

and we multiply the discrete equation (2.5) by $\varphi_{i}^{n}$. After summing over $i$ and $n$, and then applying summation by parts we obtain the identity

$$
\begin{aligned}
& \Delta x \Delta t \sum_{n=1}^{\infty} \sum_{i=-\infty}^{\infty} u_{i}^{n} \frac{\varphi_{i}^{n}-\varphi_{i}^{n-1}}{\Delta t} \\
& -\Delta t \sum_{n=0}^{\infty} \sum_{i=-\infty}^{\infty}\left(M_{i+1 / 2}^{n,-}+M_{i+1 / 2}^{n,+}\right)\left(\varphi_{i+1 / 2}^{n}+O(\Delta x)\right)=0 .
\end{aligned}
$$

We want to prove that equation (2.12) can be obtained by passing to the limit as $\Delta$ tends to 0 in (2.13). The convergence of the first term in (2.13) to the corresponding one in (2.12) is obtained as in the conservative case. Concerning the second term, since $M^{ \pm}$ 
are Lipschitz continuous and the states $u_{i}^{n}$ are uniformly bounded, we only have to study the convergence of

$$
\begin{aligned}
& \Delta t \sum_{n=0}^{\infty} \sum_{i=-\infty}^{\infty}\left(M_{i+1 / 2}^{n,-}+M_{i+1 / 2}^{n,+}\right) \varphi_{i+1 / 2}^{n} \\
& \quad=\Delta t \sum_{n=0}^{\infty} \sum_{i=-\infty}^{\infty}\left(\int_{0}^{1} A\left(\Phi\left(s ; u_{i}^{n}, u_{i+1}^{n}\right)\right) \Phi_{s}\left(s ; u_{i}^{n}, u_{i+1}^{n}\right) d s\right) \varphi_{i+1 / 2}^{n},
\end{aligned}
$$

where we have used our assumption that the scheme is formally consistent with the given family of paths $\Phi$.

Using the definition of the product $A\left(u^{\Delta}(t, \cdot)\right) u_{x}^{\Delta}(t, \cdot)$ as a measure this term can be rewritten as follows:

$$
\begin{aligned}
& \int_{0}^{\infty}\left\langle\left[A\left(u^{\Delta}(t, \cdot)\right) u_{x}^{\Delta}(t, \cdot)\right]_{\Phi}, \varphi(t, \cdot)\right\rangle d t \\
& +\sum_{n=0}^{\infty} \sum_{i=-\infty}^{\infty} \int_{t_{n}}^{t_{n+1}}\left(\int_{0}^{1} A\left(\Phi\left(s ; u_{i}^{n}, u_{i+1}^{n}\right)\right) \Phi_{s}\left(s ; u_{i}^{n}, u_{i+1}^{n}\right) d s\right)\left(\varphi_{i+1 / 2}^{n}-\varphi\left(t, x_{i+1 / 2}\right)\right) d t
\end{aligned}
$$

The second summand trivially converges to 0 . The proof is concluded by using the main stability result and error estimate in [13]. Indeed, if the sequence $u^{\Delta}$ converges in the sense of graphs, then, as recalled in (2.4) the nonconservative product converges in the weak-star sense of measures,

$$
\int_{0}^{\infty}\left\langle\left[A\left(u^{\Delta}(t, \cdot)\right) u_{x}^{\Delta}(t, \cdot)\right]_{\Phi}, \varphi(t, \cdot)\right\rangle d t \longrightarrow \int_{0}^{\infty}\left\langle\left[A(v(t, \cdot)) v_{x}(t, \cdot)\right]_{\Phi}, \varphi(t, \cdot)\right\rangle d t
$$

and we recover the exact system (2.11). In the general case, the $\Phi$-graph completion $\left(X^{\Delta}, U^{\Delta}\right)$ of $u^{\Delta}$ converges to some limiting graph $(\bar{X}, \bar{U})$,

$$
\left(X^{\Delta}, U^{\Delta}\right) \rightarrow(\bar{X}, \bar{U})
$$

which is such that its projected BV function coincides with the pointwise limit $v$ of $u^{\Delta}$. In turn the limit nonconservative product is based on the BV function $v$ and we obtain

$$
\begin{aligned}
& \int_{0}^{\infty}\left\langle\left[A\left(u^{\Delta}(t, \cdot)\right) u_{x}^{\Delta}(t, \cdot)\right]_{\Phi}, \varphi(t, \cdot)\right\rangle d t \\
& \left.\longrightarrow \int_{0}^{\infty} \int_{\mathbb{R}} A(\bar{U}(t, \cdot)) \bar{U}(t, \cdot) \varphi(t, \cdot)\right\rangle d t=\int_{0}^{\infty}\left\langle\left[A(v(t, \cdot)) v_{x}(t, \cdot)\right]_{\Phi}+\mu_{v}(t), \varphi(t, \cdot)\right\rangle d t
\end{aligned}
$$

for some time-dependent measure $\mu_{v}$. This completes the proof.

\section{Equivalent equations for nonconservative systems}

\subsection{Derivation of the equivalent equation}

In this section, we derive the equivalent equations corresponding to the Lax-Friedrichstype scheme introduced in Subsection 2.2, which reads

$$
\begin{aligned}
\frac{1}{\Delta t}\left(u_{i}^{n+1}-\frac{1}{2}\left(u_{i-1}^{n}+u_{i+1}^{n}\right)\right)+\frac{1}{2 \Delta x}( & \int_{0}^{1} A\left(\Phi\left(s ; u_{i-1}^{n}, u_{i}^{n}\right)\right) \frac{\partial \Phi}{\partial s}\left(s ; u_{i-1}^{n}, u_{i}^{n}\right) d s \\
& \left.+\int_{0}^{1} A\left(\Phi\left(s ; u_{i}^{n}, u_{i+1}^{n}\right)\right) \frac{\partial \Phi}{\partial s}\left(s ; u_{i}^{n}, u_{i+1}^{n}\right) d s\right)=0 .
\end{aligned}
$$


We consider first the equivalent equation at second order. Performing a formal Taylor expansion in the scheme we obtain:

$$
\begin{aligned}
& v_{t}+A(v) v_{x}+\frac{\Delta t}{2}\left(v_{t t}-\frac{\Delta x^{2}}{\Delta t^{2}} v_{x x}+\frac{\Delta x}{\Delta t} I_{2}(v)\right)=0 \\
& I_{2}(v)=\int_{0}^{1} D A(v)\left(D_{u_{l}} \Phi \cdot v_{x}, D_{u_{l}} \Phi_{s} \cdot v_{x}\right) d s+\int_{0}^{1} D A(v)\left(D_{u_{r}} \Phi \cdot v_{x}, D_{u_{r}} \Phi_{s} \cdot v_{x}\right) d s
\end{aligned}
$$

where the following notation has been used: given two vectors $v=\left[v_{1}, \ldots, v_{N}\right]^{T}, w=$ $\left[w_{1}, \ldots, w_{N}\right]^{T}, D A(u)(v, w)$ represents the derivative of $A(u)$ in the direction of the vector $v$ (which is a matrix) applied to the vector $w$, i.e. $D A(u)(v, w)=\left(\sum_{k=1}^{N} v_{k} \partial_{u_{k}} A(u)\right) \cdot w$, where $\partial_{u_{k}} A(u)$ is the $N \times N$ matrix whose $(i, j)$ entry is $\partial_{u_{k}} a_{i, j}(u)$.

As usual, we write the $t$-derivatives using $x$-derivatives in order to obtain a new modified equation. Using the equality

$$
\left(A^{2}(v) v_{x}\right)_{x}=D A(v)\left(v_{x}, A(v) v_{x}\right)+A(v)\left(A(v) v_{x}\right)_{x},
$$

we obtain the modified equation for the Lax-Friedrichs method:

$$
\begin{aligned}
v_{t}+A(v) v_{x}= & \frac{\Delta x^{2}}{2 \Delta t}\left(v_{x x}-\frac{\Delta t^{2}}{\Delta x^{2}}\left(A^{2}(v) v_{x}\right)_{x}\right. \\
& \left.-\frac{\Delta t^{2}}{\Delta x^{2}}\left(D A(v)\left(A(v) v_{x}, v_{x}\right)-D A(v)\left(v_{x}, A(v) v_{x}\right)\right)\right)-\frac{\Delta x}{2} I_{2}(v) .
\end{aligned}
$$

Note that, in the expression of the modified equations, the only term that depends on the choice of the family of paths is the last one: $\Phi$ only appears in the expression of $I_{2}(v)$.

In a similar way, by a simple (but tedious) calculation we can obtain the equivalent equations at third order:

$$
\begin{aligned}
v_{t} & +A(v) v_{x} \\
= & \Delta x\left(\frac{\Delta x}{2 \Delta t} v_{x x}-\frac{\Delta t}{2 \Delta x} \Theta(v)-\frac{1}{2} I_{2}(v)\right)+\Delta x^{2}\left(\frac{1}{2}\left(A(v) v_{x x}\right)_{x}+\frac{1}{6} A(v) v_{x x x}\right. \\
& -\frac{\Delta t^{2}}{3 \Delta x^{2}}\left((A(v) \Theta(v))_{x}-D A(v)\left(\Theta(v), v_{x}\right)-D A(v)\left(v_{x}, \Theta(v)\right)\right) \\
& -\frac{\Delta t^{2}}{12 \Delta x^{2}} D^{2} A(v)\left(A(v) v_{x}, A(v) v_{x}, v_{x}\right)-\frac{\Delta t^{2}}{6 \Delta x^{2}} D A(v)\left(A(v) v_{x},\left(A(v) v_{x}\right)_{x}\right) \\
& -\frac{\Delta t}{4 \Delta x}\left(\left(A(v) I_{2}(v)\right)_{x}+D A(v)\left(I_{2}(v), v_{x}\right)+D A(v)\left(v_{x}, I_{2}(v)\right)\right) \\
& \left.-\frac{1}{4} D^{2} A(v)\left(v_{x}, v_{x}, v_{x}\right)-\frac{1}{4} I_{3}(v)-\frac{\Delta t}{4 \Delta x}\left(I_{2}(v)\right)_{t}\right),
\end{aligned}
$$

where we have used the notation

$$
D^{2} A(u)\left(v^{1}, v^{2}, w\right)=\left(\sum_{k, m=1}^{N} v_{k}^{1} v_{m}^{2} \partial_{u_{k} u_{m}}^{2} A(u)\right) \cdot w
$$

and

$$
\Theta(v)=\left(A^{2}(v) v_{x}\right)_{x}+D A(v)\left(A(v) v_{x}, v_{x}\right)-D A(v)\left(v_{x}, A(v) v_{x}\right) .
$$

Furthermore, the expansion of $\left(I_{2}(v)\right)_{t}$ is

$$
\left(I_{2}(v)\right)_{t}=I_{2,1}(v)+I_{2,2}(v)+I_{2,3}(v),
$$


with

$$
\begin{gathered}
I_{2,1}(v)=\int_{0}^{1} D^{2} A(v)\left(A(v) v_{x}, D_{u_{l}} \Phi \cdot v_{x}, D_{u_{l}} \Phi_{s} \cdot v_{x}\right) d s \\
\quad+\int_{0}^{1} D^{2} A(v)\left(A(v) v_{x}, D_{u_{r}} \Phi \cdot v_{x}, D_{u_{r}} \Phi_{s} \cdot v_{x}\right) d s \\
\quad+\int_{0}^{1} D A(v)\left(D_{u_{l} u_{l}}^{2} \Phi\left(A(v) v_{x}, v_{x}\right), D_{u_{l}} \Phi_{s} \cdot v_{x}\right) d s \\
\quad+\int_{0}^{1} D A(v)\left(D_{v_{r} v_{r}}^{2} \Phi\left(A(v) v_{x}, v_{x}\right), D_{u_{r}} \Phi_{s} \cdot v_{x}\right) d s, \\
I_{2,2}(v)=\int_{0}^{1} D A(v)\left(D_{u_{l}} \Phi \cdot\left(D A(v)\left(v_{x}, v_{x}\right)+A(v) v_{x x}\right), D_{u_{l}} \Phi_{s} \cdot v_{x}\right) d s \\
\quad+\int_{0}^{1} D A(v)\left(D_{u_{r}} \Phi \cdot\left(D A(v)\left(v_{x}, v_{x}\right)+A(v) v_{x x}\right), D_{u_{r}} \Phi_{s} \cdot v_{x}\right) d s \\
\quad+\int_{0}^{1} D A(v)\left(D_{u_{l}} \Phi \cdot v_{x}, D_{u_{l} u_{l}}^{2} \Phi_{s}\left(A(v) v_{x}, v_{x}\right)\right) d s \\
\quad+\int_{0}^{1} D A(v)\left(D_{u_{r}} \Phi \cdot v_{x}, D_{u_{r} u_{r}}^{2} \Phi_{s}\left(A(v) v_{x}, v_{x}\right)\right) d s,
\end{gathered}
$$

and

$$
\begin{aligned}
I_{2,3}(v)= & \int_{0}^{1} D A(v)\left(D_{u_{l}} \Phi \cdot v_{x}, D_{u_{l}} \Phi_{s} \cdot\left(D A(v)\left(v_{x}, v_{x}\right)+A(v) v_{x x}\right)\right) d s \\
& +\int_{0}^{1} D A(v)\left(D_{u_{r}} \Phi \cdot v_{x}, D_{u_{r}} \Phi_{s} \cdot\left(D A(v)\left(v_{x}, v_{x}\right)+A(v) v_{x x}\right)\right) d s
\end{aligned}
$$

The above formula illustrate the high complexity of the equivalent equation approach when dealing with nonconservative schemes.

\subsection{Role of the equivalent equation}

These modified equations are useful to understand why the numerical solutions may not converge to the weak solutions of the system. Let us suppose that the family of paths $\Phi$ used in the definition of weak solutions is based on a parabolic regularization of the system

$$
u_{t}^{\epsilon}+A\left(u^{\epsilon}\right) u_{x}^{\epsilon}=\epsilon\left(D\left(u^{\epsilon}\right) u_{x}^{\epsilon}\right)_{x}
$$

where $D$ is a viscosity matrix which is admissible in the following sense: if $u_{l}, u_{r}$ can be connected by a discontinuity satisfying the Rankine-Hugoniot conditions related to the family of paths:

$$
\xi\left(u_{r}-u_{l}\right)=\int_{0}^{1} A\left(\Phi\left(s ; u_{l}, u_{r}\right)\right) \frac{\partial \Phi}{\partial s}\left(s ; u_{l}, u_{r}\right) d x
$$

for some $\xi \in \mathbb{R}$, then $\Phi\left(s ; u_{l}, u_{r}\right)$ is a reparametrization of the solution of the differential system:

with the conditions:

$$
-\xi v^{\prime}+A(v) v^{\prime}=\left(D(v) v^{\prime}\right)^{\prime},
$$

$$
\lim _{\xi \rightarrow-\infty} v(\xi)=u_{l}, \quad \lim _{\xi \rightarrow \infty} v(\xi)=u_{r} .
$$


Under these hypotheses it can be checked [19] that the function

$$
u(x, t)= \begin{cases}u_{l} & x<\xi t, \\ u_{r} & x>\xi t .\end{cases}
$$

is a weak solution in the sense of Dal Maso, LeFloch, and Murat.

If now the Lax-Friedrichs scheme is applied to the hyperbolic system, the limits of the numerical solutions provide approximations to the vanishing viscosity limits related to the regularization (3.1) which is different of (3.2). The difficulty comes from the fact that, unlike the conservative case, the vanishing viscosity limits depend on the regularization of the problem. Even if, for simplicity, we have only calculated the modified equations corresponding to the Lax-Friedrichs scheme, the same difficulty would be present for any other scheme involving a numerical viscous term: the numerical solutions approximate the vanishing viscosity limit of a modified equation whose regularization terms depend both on the chosen family of paths and on the specific form of its viscous terms.

\section{Examples of nonconservative hyperbolic systems}

\subsection{A simplified model}

We begin with a hyperbolic system containing nonconservative products, which will be used in the following section to perform numerical experiments. We consider the system

$$
\begin{aligned}
& h_{t}+q_{x}=0, \\
& q_{t}+\left(\frac{q^{2}}{h}\right)_{x}+q h h_{x}=0,
\end{aligned}
$$

which has the form $w_{t}+A(w) w_{x}=0$ with

$$
w=\left[\begin{array}{l}
h \\
q
\end{array}\right], \quad A(w)=\left[\begin{array}{cc}
0 & 1 \\
-u^{2}+u^{2} h & 2 u
\end{array}\right],
$$

and $u=q / h$. In the region

$$
\Omega=\left\{(h, q) \mid 0<q, 0<h<(16 q)^{1 / 3}\right\},
$$

the system is strictly hyperbolic and all characteristic fields are genuinely nonlinear. The eigenvalues of this system are

$$
\lambda_{1}=u-h \sqrt{u}, \quad \lambda_{2}=u+h \sqrt{u},
$$

and the integral curves of the first and second characteristic fields are

$$
\sqrt{u}+h / 2=\text { const }, \quad \sqrt{u}-h / 2=\text { const },
$$

respectively.

In order to define the jump conditions, the paths connecting the left- and right-hand limits $w^{ \pm}=\left[h^{ \pm}, q^{ \pm}\right]$at a shock are chosen to be the union of the segment connecting $w^{-}$with $w^{*}=\left[h^{+}, q^{-}\right]$and the segment connecting $w^{*}$ to $w^{+}$: 


$$
\Phi\left(s ; w^{-}, w^{+}\right)=\left\{\begin{array}{cc}
{\left[\begin{array}{c}
h^{-}+2 s\left(h^{+}-h^{-}\right) \\
q^{-}
\end{array}\right],} & 0 \leq s \leq 1 / 2 ; \\
{\left[\begin{array}{c}
h^{+} \\
q^{-}+(2 s-1)\left(q^{+}-q^{-}\right)
\end{array}\right],} & 1 / 2 \leq s \leq 1 ;
\end{array}\right.
$$

Such paths were introduced in [13] to illustrate certain issues encountered with nonconservative products. The corresponding jump conditions (3.3) are the following:

$$
\begin{aligned}
& \xi[h]=[q], \\
& \xi[q]=\left[\frac{q^{2}}{h}\right]+q^{-}\left[\frac{h^{2}}{2}\right] .
\end{aligned}
$$

Once the jump conditions have been stated, it is possible to solve the Riemann problem for any pair of states which are sufficiently enough. Then, a family of paths satisfying (R2) and (R3) is constructed. In Figure 1 the path linking the states $w_{l}=[1,1]^{T}$ and $w_{r}=[0.5,0.5]^{T}$ is depicted. In this case, the solution of the Riemann problem consists of a 1-rarefaction connecting $w_{l}$ to an intermediate state $\tilde{w}$ and a 2 -shock linking $\tilde{w}$ to $w_{r}$. As a consequence, the path consists of the arc of the 1-integral curve linking $w_{l}$ to $\tilde{w}$ and two segments connecting $\tilde{w}$ and $w_{r}$ which are parallel to the axis. The set of states that can be connected to $w_{l}$ by an entropy satisfying 1 -wave or 2 -wave are also depicted. In each case $R i$ denotes $i$-rarefactions and $S i$ denotes $i$-shocks.

\subsection{A class of systems of balance laws}

We consider PDE systems of the form:

$$
w_{t}+F(w)_{x}=S(w) \sigma_{x},
$$

where the unknown $w(x, t)$ takes values on an open convex set $\mathcal{O}$ of $\mathbb{R}^{N} ; F$ and $S$ are regular functions from $\mathcal{O}$ to $\mathbb{R}^{N}$; and $\sigma(x)$ is a known function from $\mathbb{R}$ to $\mathbb{R}$. For an account of the existing literature on well-balanced schemes for this class of systems we refer to the lecture notes by Bouchut [4], as well as to the recent contribution by Noelle et al. [29], and Xin and Shu [33].

As pointed out in LeFloch [19] for the Euler equations in nozzle with discontinuous cross-section, such a system can be recast in the form of a nonconservative system $W_{t}+$ $A(W) W_{x}=0$, by introducing

$$
W=\left[\begin{array}{c}
w \\
\sigma
\end{array}\right], \quad A(W)=\left[\begin{array}{c|c}
J(w) & -S(w) \\
\hline 0 & 0
\end{array}\right],
$$

where $J(w)$ denotes the Jacobian matrix of $F$ :

$$
J(w)=\frac{\partial F}{\partial w}(w)
$$




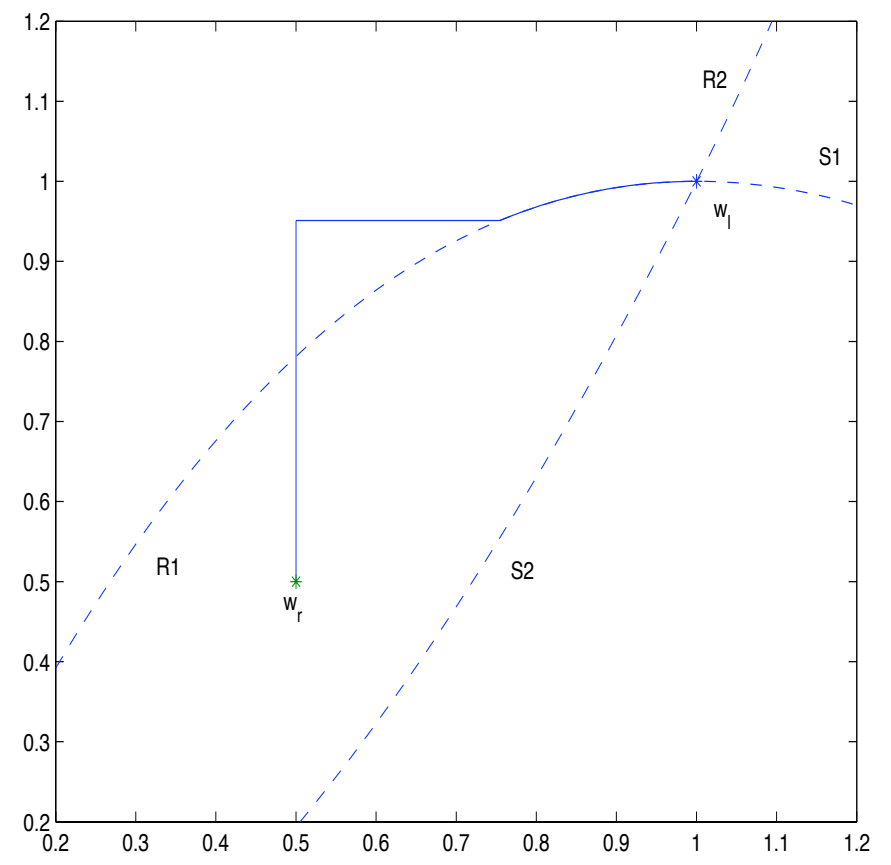

Fig. 1. Path connecting the states $w_{l}$ and $w_{r}$ (continuous line) and set of states that can be connected to $w_{l}$ by an entropy satisfying 1 or 2 -wave (dashed lines).

If $J$ has $N$ different real and non-vanishing eigenvalues $\lambda_{1}(w), \ldots, \lambda_{N}(w)$, then the system is strictly hyperbolic with eigenvalues

$$
\lambda_{1}(w), \ldots, \lambda_{N}(w), 0 .
$$

Clearly, the $(N+1)$-th field is linearly degenerate and, for definiteness, we may assume that all other fields are genuinely nonlinear.

In order to define weak solutions to this nonconservative system, a family of paths

$$
\Phi\left(s ; W_{l}, W_{r}\right)=\left[\begin{array}{c}
\Phi_{w}\left(s ; W_{l}, W_{r}\right) \\
\Phi_{\sigma}\left(s ; W_{l}, W_{r}\right)
\end{array}\right]
$$

must be chosen and it is natural to impose the following requirement: if $W=[w, \sigma]^{T}$ is a weak solution to the system and $\sigma$ is a constant, then $w$ must be a weak solution of the system of conservation laws:

$$
w_{t}+F(w)_{x}=0 .
$$

This requirement is satisfied if the family of paths fulfills the following condition: (R4) If $W_{l}$ and $W_{r}$ are such that $\sigma_{l}=\sigma_{r}=\bar{\sigma}$, then:

$$
\Phi_{\sigma}\left(s ; W_{l}, W_{r}\right)=\bar{\sigma}, \quad s \in[0,1] .
$$


In fact any family of paths satisfying (R4) together with the requirements (R1) and (R3) (cf. Section 2.2), leads to the same notion of weak solution for such systems. These solutions contain two type of discontinuities :

- Shock waves across which $\sigma$ is continuous that satisfy the usual Rankine-Hugoniot conditions:

$$
\xi\left(w^{+}-w^{-}\right)=F\left(w^{+}\right)-F\left(w^{-}\right) .
$$

- Stationary contact discontinuities placed at the jumps of $\sigma$ and connecting two states that belong to the same integral curve of the linearly degenerate field.

For these systems, the difficulties of convergence commented above are not appreciated for the finite difference schemes introduced in Section 2.2. Moreover, the shock waves propagating in regions where $\sigma$ is continuous are correctly captured independently of the choice of paths. Nevertheless, in order to correctly capture the stationary contact discontinuities related to the jumps of $\sigma$, the numerical schemes have to be based on families of paths that satisfy at least the requirement $(\mathrm{R} 1)$. Interestingly, this is also the requirement necessary to obtain well-balanced schemes.

The Lax-Friedrichs scheme presented in Section 2.2 cannot be used as it stands for systems of balance laws as it does not preserve the equation

$$
\sigma_{t}=0
$$

In effect, it can be easily verified that the numerical scheme for the variable $\sigma$ reads as follows:

$$
\sigma_{i}^{n+1}=\frac{\sigma_{i-1}^{n}+\sigma_{i+1}^{n}}{2} .
$$

In [10] the following modification of the scheme was introduced to get rid of this difficulty: instead of (2.9), $M_{i+1 / 2}^{n, \pm}$ are given by

$$
\begin{aligned}
& M_{i+1 / 2}^{n,-}=\widehat{A}_{i+1 / 2}^{n,-} \cdot\left(u_{i+1}^{n}-u_{i}^{n}\right), \\
& M_{i+1 / 2}^{n,+}=\widehat{A}_{i+1 / 2}^{n,+} \cdot\left(u_{i+1}^{n}-u_{i}^{n}\right),
\end{aligned}
$$

where

$$
\widehat{A}_{i+1 / 2}^{n, \pm}=\frac{1}{2}\left( \pm \frac{\Delta x}{\Delta t} \widehat{I}_{i+1 / 2}^{n}+A_{i+1 / 2}^{n}\right) .
$$

Here, $A_{i+1 / 2}^{n}$ is a Roe linearization and

$$
\widehat{I}_{i+1 / 2}^{n}=\mathcal{K}_{i+1 / 2}^{n} \cdot \widehat{I d} \cdot\left(\mathcal{K}_{i+1 / 2}^{n}\right)^{-1},
$$

where $\mathcal{K}_{i+1 / 2}^{n}$ is a matrix whose columns are eigenvectors of $A_{i+1 / 2}^{n}$ associated to $\lambda_{i+1 / 2,1}^{n}$, $\ldots, \lambda_{i+1 / 2, N}^{n}$, and $\widehat{I d}$ is the diagonal matrix whose $j$-th coefficient is 1 if $\lambda_{i+1 / 2, j}^{n} \neq 0$, or 0 if $\lambda_{i+1 / 2, j}^{n}=0$. Note that if, instead, $\widehat{I}_{i+1 / 2}^{n}$ is taken to be equal to the identity matrix, then the Lax-Friedrichs scheme presented in Section 2.2 is recovered.

An important particular example of systems of balance laws is the shallow water system governing the flow of a shallow layer of inviscid homogeneous fluid through a straight channel with a constant rectangular cross-section:

$$
\begin{aligned}
& \frac{\partial h}{\partial t}+\frac{\partial q}{\partial x}=0 \\
& \frac{\partial q}{\partial t}+\frac{\partial}{\partial x}\left(\frac{q^{2}}{h}+\frac{g}{2} h^{2}\right)=g h \frac{\mathrm{d} H}{\mathrm{~d} x} .
\end{aligned}
$$


The variable $x$ makes reference to the axis of the channel and $t$ is time; $q(x, t)$ and $h(x, t)$ represent the mass-flow and the thickness, respectively; $g$, the gravity; and $H(x)$, the depth measured from a fixed level of reference.

The eigenvalues of this matrix are $\lambda_{1}=u-c, \lambda_{2}=u+c$, and 0 , where $c=\sqrt{g h}$. In this case, the equations of the integral curves of the linearly degenerate field are given by the equations

$$
q=\text { const }, \quad h+\frac{q^{2}}{2 g h^{2}}-H=\text { const } .
$$

The resonant regime where $\lambda_{1}$ or $\lambda_{2}$ vanish are not considered in the present paper; for a discussion of the Riemann problem see LeFloch and Thanh [27].

\subsection{Two-layer shallow water system}

We consider in this paragraph the system of partial differential equations governing the one-dimensional flow of two superposed immiscible layers of shallow water fluids over a flat bottom topography (see [8] for details) :

$$
\begin{aligned}
& \frac{\partial h_{1}}{\partial t}+\frac{\partial q_{1}}{\partial x}=0, \\
& \frac{\partial q_{1}}{\partial t}+\frac{\partial}{\partial x}\left(\frac{q_{1}^{2}}{h_{1}}+\frac{g}{2} h_{1}^{2}\right)=-g h_{1} \frac{\partial h_{2}}{\partial x}, \\
& \frac{\partial h_{2}}{\partial t}+\frac{\partial q_{2}}{\partial x}=0, \\
& \frac{\partial q_{2}}{\partial t}+\frac{\partial}{\partial x}\left(\frac{q_{2}^{2}}{h_{2}}+\frac{g}{2} h_{2}^{2}\right)=-\frac{\rho_{1}}{\rho_{2}} g h_{2} \frac{\partial h_{1}}{\partial x} .
\end{aligned}
$$

In these equations, the index 1 refers to the upper layer and the index 2 to the lower one. The fluid is assumed to occupy a straight channel with constant rectangular cross-section and constant width. The coordinate $x$ refers to the axis of the channel, $t$ denotes the time variable, and $g$ is the gravity. Each layer is assumed to have a constant density $\rho_{i}, i=1,2$ $\left(\rho_{1}<\rho_{2}\right)$, while the unknowns $q_{i}(x, t)$ and $h_{i}(x, t)$ represent respectively the mass-flow and the thickness of the $i$-th layer at the section of coordinate $x$ at time $t$.

System (4.7) can be written in the form (1.1), say $w_{t}+A(w) w_{x}=0, w=w(t, x) \in \mathbb{R}^{N}$, with $N=4$ and

$$
w=\left[\begin{array}{c}
h_{1} \\
q_{1} \\
h_{2} \\
q_{2}
\end{array}\right], \quad A(w)=\left[\begin{array}{cccc}
0 & 1 & 0 & 0 \\
-u_{1}^{2}+c_{1}^{2} & 2 u_{1} & c_{1}^{2} & 0 \\
0 & 0 & 0 & 1 \\
r c_{2}^{2} & 0 & -u_{2}^{2}+c_{2}^{2} & 2 u_{2}
\end{array}\right],
$$

where $u_{i}=q_{i} / h_{i}$ represents the averaged velocity of the $i$-th layer, $c_{i}=\sqrt{g h_{i}}, i=1,2$, and $r=\frac{\rho_{1}}{\rho_{2}}$. The characteristic equation of the system is

$$
\left(\lambda^{2}-2 u_{1} \lambda+u_{1}^{2}-g h_{1}\right)\left(\lambda^{2}-2 u_{2} \lambda+u_{2}^{2}-g h_{2}\right)=r g^{2} h_{1} h_{2} .
$$

Observe that, when $r=0$, the eigenvalues are those corresponding to each layer separately. In this situation, the coupling terms do not affect the nature of the system in any essential manner. 
In the case $r \cong 1$ (which is the situation arising in many geophysical flows) a first-order approximation of the eigenvalues was given in [32]:

$$
\begin{aligned}
& \lambda_{\text {ext }}^{ \pm} \cong \frac{u_{1} h_{1}+u_{2} h_{2}}{h_{1}+h_{2}} \pm\left(g\left(h_{1}+h_{2}\right)\right)^{1 / 2} \\
& \lambda_{\text {int }}^{ \pm} \cong \frac{u_{1} h_{2}+u_{2} h_{1}}{h_{1}+h_{2}} \pm\left(g^{\prime} \frac{h_{1} h_{2}}{\left(h_{1}+h_{2}\right)}\left(1-\frac{\left(u_{1}-u_{2}\right)^{2}}{g^{\prime}\left(h_{1}+h_{2}\right)}\right)\right)^{1 / 2} .
\end{aligned}
$$

In the former expression, $g^{\prime}=(1-r) g$ is the reduced gravity.

It is not easy to check the genuinely nonlinear character of the 4 characteristic fields, as the eigenvalues and eigenvectors can not be written explicitely in a simple manner. Nevertheless, this fact is easily proved in the case $r=0$ as, in this case, the system reduces to two decoupled shallow water systems. As a consequence, using a continuity argument, this is also true at least for small values of $r$.

From equation (4.9) we can observe that the internal eigenvalues may become complex. This situation occurs when they satisfy, approximately, the following inequality:

$$
\frac{\left(u_{1}-u_{2}\right)^{2}}{g^{\prime}\left(h_{1}+h_{2}\right)}>1 \text {. }
$$

In this case, the system loses its hyperbolic character. These situations are related with the appearance of shear instabilities that may lead, in real flows, to intense mixing of the two layers. While, in practice, this mixture partially dissipates the energy, in numerical experiments these interface disturbances grow and overwhelm the solution. Clearly, we cannot expect to simulate these phenomena with a two-immiscible-layer model. Therefore, the above inequality in fact gives the range of validity of a model based on the equations (4.7), if viscosity effects are neglected. In this work only the case where the matrix $A(w)$ has real eigenvalues is considered, i.e. the system is supposed to be strictly hyperbolic.

The jump conditions (3.3) related to the choice of a family of paths

$$
\Phi\left(s ; w_{l}, w_{r}\right)=\left[\begin{array}{c}
\Phi_{h_{1}}\left(s ; w_{l}, w_{r}\right) \\
\Phi_{q_{1}}\left(s ; w_{l}, w_{r}\right) \\
\Phi_{h_{2}}\left(s ; w_{l}, w_{r}\right) \\
\Phi_{q_{2}}\left(s ; w_{l}, w_{r}\right)
\end{array}\right]
$$

read 


$$
\begin{aligned}
\xi\left(h_{1}^{r}-h_{1}^{l}\right)= & q_{1}^{r}-q_{1}^{l}, \\
\xi\left(q_{1}^{r}-q_{1}^{l}\right)= & \frac{\left(q_{1}^{r}\right)^{2}}{h_{1}^{r}}-\frac{\left(q_{1}^{l}\right)^{2}}{h_{1}^{l}}+\frac{g}{2}\left(h_{1}^{r}\right)^{2}-\frac{g}{2}\left(h_{1}^{l}\right)^{2} \\
& +g \int_{0}^{1} \Phi_{h_{1}}\left(s ; w_{l}, w_{r}\right) \frac{\partial \Phi_{h_{2}}}{\partial s}\left(s ; w_{l}, w_{r}\right) d s, \\
\xi\left(h_{2}^{r}-h_{2}^{l}\right)= & q_{2}^{r}-q_{2}^{l}, \\
\xi\left(q_{2}^{r}-q_{2}^{l}\right)= & \frac{\left(q_{2}^{r}\right)^{2}}{h_{2}^{r}}-\frac{\left(q_{2}^{l}\right)^{2}}{h_{2}^{l}}+\frac{g}{2}\left(h_{2}^{r}\right)^{2}-\frac{g}{2}\left(h_{2}^{l}\right)^{2} \\
& +g r \int_{0}^{1} \Phi_{h_{2}}\left(s ; w_{l}, w_{r}\right) \frac{\partial \Phi_{h_{1}}}{\partial s}\left(s ; w_{l}, w_{r}\right) d s .
\end{aligned}
$$

Observe that these conditions are independent of the choice of $\Phi_{q_{i}}\left(s: w_{l}, w_{r}\right), i=1,2$.

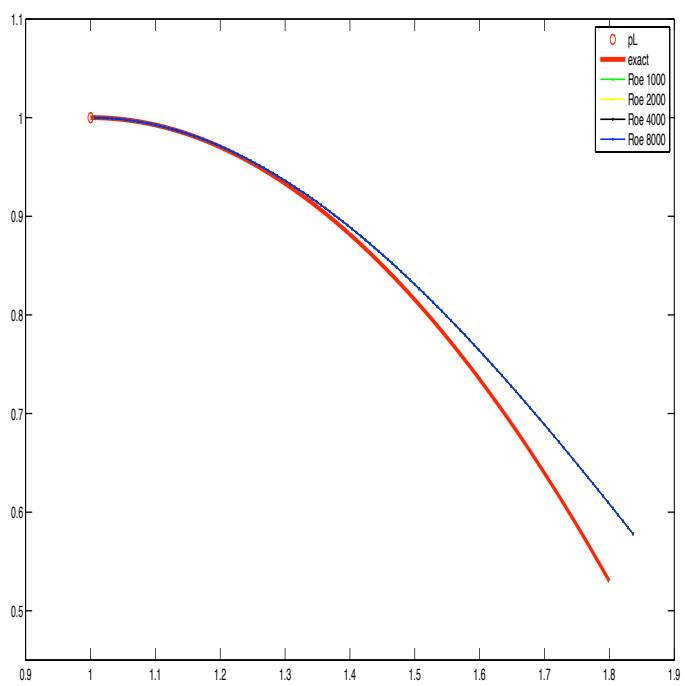

Fig. 2. Test case 5.1. Hugoniot curves: exact (continuous red line) and numerical (line with dots) obtained with Roe scheme

\section{Numerical experiments}

\subsection{A simplified system}

We will demonstrate here that, in general, a difference scheme for a general nonconservative hyperbolic system (1.1) does not converge to the exact solution $u$, that is, with the notation already introduced in previous sections we claim that

$$
v=\lim _{\Delta \rightarrow 0} u^{\Delta} \neq u \text {. }
$$


This fact was first observed for nonconservative schemes for scalar equations in Hou and LeFloch [16] and, in the context of nonclassical shocks generated by diffusion and dispersion, in Hayes and LeFloch [15,25]. Here, we observe (5.1) for finite difference approximations of nonconservative systems.

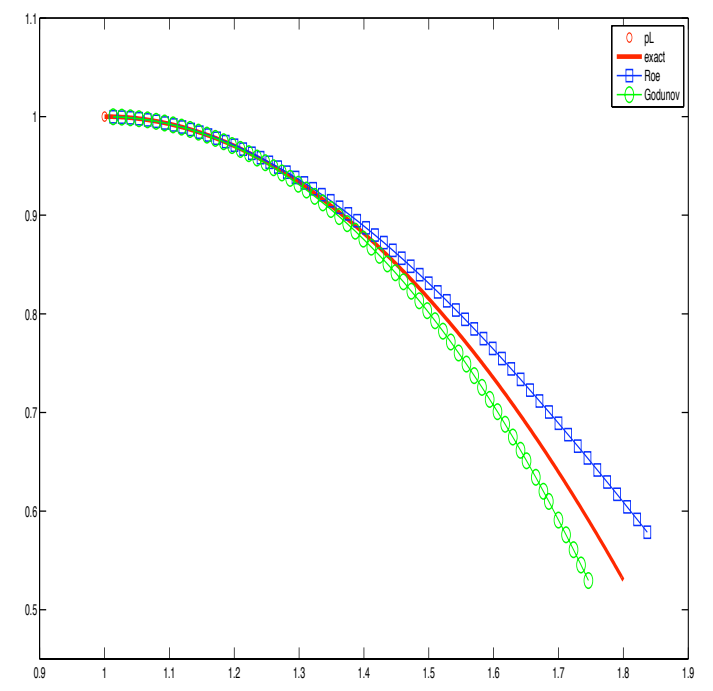

Fig. 3. Test case 5.1. Hugoniot curves: exact (continuous red line), Roe (blue line with squares) and Godunov (green line with circles)

We begin with the convergence of Roe and Godunov methods for (4.1). The Roe method considered here is consistent with the family of paths given by (4.2) for every pair of states. The corresponding Roe matrix is as follows:

$$
A_{i+1 / 2}^{n}=\left[\begin{array}{ccc}
0 & 1 \\
-\left(u_{i+1 / 2}^{n}\right)^{2}+q_{i}^{n} h_{i+1 / 2}^{n} & 2 u_{i+1 / 2}^{n}
\end{array}\right],
$$

where

$$
u_{i+1 / 2}^{n}=\frac{\sqrt{h_{i}^{n}} u_{i}^{n}+\sqrt{h_{i+1}^{n}} u_{i+1}^{n}}{\sqrt{h_{i}^{n}}+\sqrt{h_{i+1}^{n}}}, \quad h_{i+1 / 2}^{n}=\frac{1}{2}\left(h_{i}^{n}+h_{i+1}^{n}\right) .
$$

On the other hand, the Godunov method considered here is based on the family of paths described in Section 4.1: it satisfies (R2)-(R3) and coincides with (4.2) for pair of states that can be linked by a shock.

We consider the Rankine-Hugoniot curve composed by the states $w_{r}$ that can connected with $w_{l}=[1 ; 1]^{T}$ by a 1 -shock, which is given by:

$$
q_{r}=h_{r}\left(1-\sqrt{\frac{h_{r}+1}{2 h_{r}}}\left(h_{r}-1\right)\right) .
$$

Figure 2 shows a plot of the curve (5.2) in the plane $h-q$ (continuous red line). 


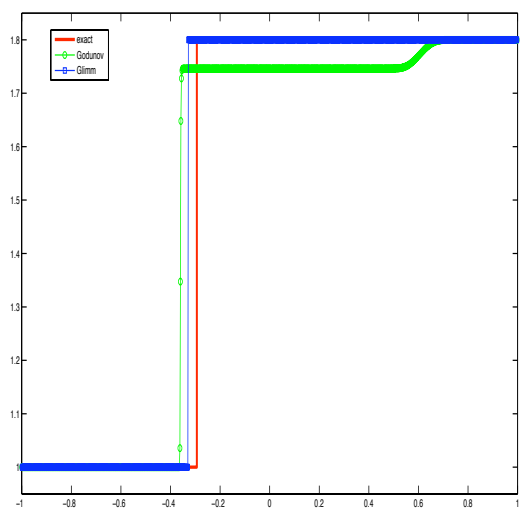

(a) h at $t=0.5$

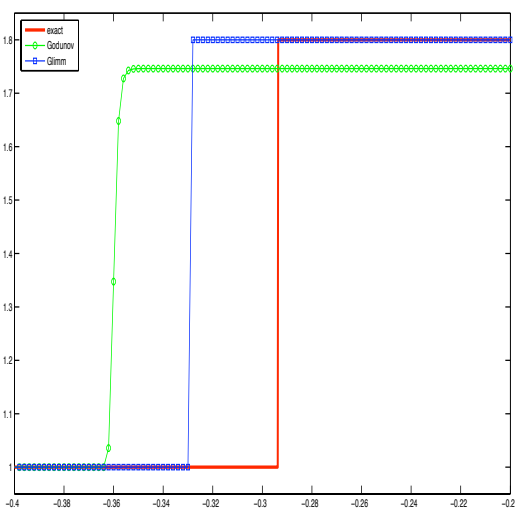

(b) h (zoom) at $t=0.5$.

Fig. 4. Test case 5.1. Solution of Riemann problem $(h)(5.3)$ at time $t=0.5$ : Exact (continuous red line), Godunov (green line with circles) and Glimm (blue line with squares)

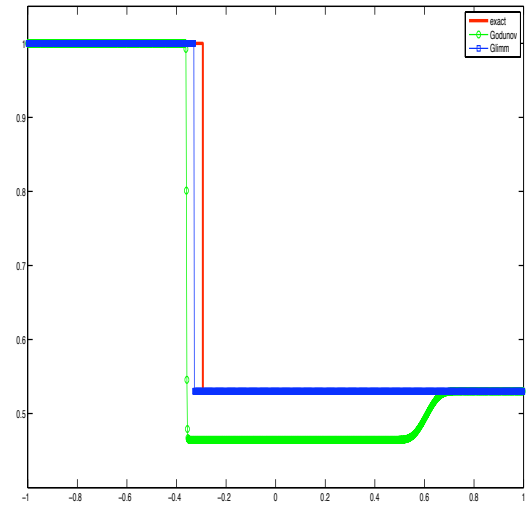

(a) q at $t=0.5$

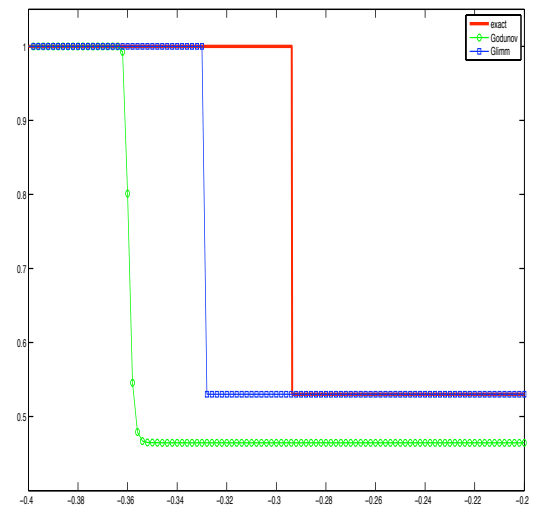

(b) q (zoom) at $t=0.5$.

Fig. 5. Test case 5.1. Solution of Riemann problem $(q)$ (5.3) at time $t=0.5$ : Exact (continuous red line), Godunov (green line with circles) and Glimm (blue line with squares)

Next, Roe method is used to solve numerically a family of Riemann problems in which the left state is $w_{l}$ and the right state $w_{r}$ runs on the Hugoniot curve (5.2). The speed of propagation and the limit states of the 1-shock are computed in the numerical solution by using the first divided difference as a smooth indicator. This computation has been performed using four meshes with decreasing mesh step $(\Delta x=0.002, \Delta x=0.001, \Delta x=$ 0.0005 and $\Delta x=0.00025)$. The CFL parameter is set to 0.9. The numerical Hugoniot 
curves obtained in this way (line with dots) are compared with the exact one (continuous red line) in Figure 2. It can be observed that the numerical Hugoniot curves converge, but the limit is not the exact one.

The same behavior is observed for Godunov method: the numerical Hugoniot-curves converge but the limit is not the exact one: in Figure 3 the exact Hugoniot curve (continuous red line) is compared with those computed with Roe (blue line with squares) and Godunov (green line with circles) methods with $\Delta x=0.001$. The CLF parameter is set to 0.9 for Roe and 0.5 for Godunov. This choice ensures that the Godunov method corresponds to advance in time by exactly solving the Riemann problems and taking the averages of the solutions at the cells.

Finally, we compare Godunov and Glimm methods. We consider a Riemann problem with initial conditions

$$
w(x, 0)= \begin{cases}w_{l}=\left(\begin{array}{l}
1 \\
1
\end{array}\right), & x<0 ; \\
w_{r}=\left(\begin{array}{c}
1.8 \\
q_{r}
\end{array}\right), & x>0 ;\end{cases}
$$

where $q_{r}$ is given by $(5.2)$ for $h_{r}=1.8\left(q_{r} \cong 0.530039370688997\right)$. The exact solution consists thus of a 1-shock linking the states. In Figures 4 and 5 the exact solution at time $t=0.5$ is compared with numerical solutions obtained with Godunov (green line with circles) and Glimm (blue line with squares) methods with $\Delta x=0.001$ and CFL $=0.5$. Note how Godunov method introduces a 2-rarefaction in the computed solution.

Observe that the first equation of (4.1) is a conservation law. According to this equation, for $A>0$ large enough, the exact solution of the Riemann problem has to satisfy the following conservation property:

$$
\int_{-A}^{A} h(x, t) d x=\int_{-A}^{A} h(x, 0) d x+t\left(1-q_{r}\right) .
$$

In Figure 6 we investigate the conservation property of both Godunov and Glimm methods: we fix $A$ and compare the exact value of the integral of $h$ at time $t_{n}$ with its numerical approximations. Note that Godunov method satisfies the conservation property exactly.

\subsection{Shallow water system}

In this section we consider the discretization of the shallow water system by means of Roe and the modified Lax-Friedrichs schemes applied to the formulation (1.1) of the problem. In the first test, we check that, for continuous bottom functions $H$, the shock waves are correctly captured for the schemes even if the numerical schemes are formally consistent with a simple family of paths. In the second one, stationary contact discontinuities placed at the jumps of $H$ are considered: we check that they are correctly captured only when the family of paths satisfies the property (R1). 


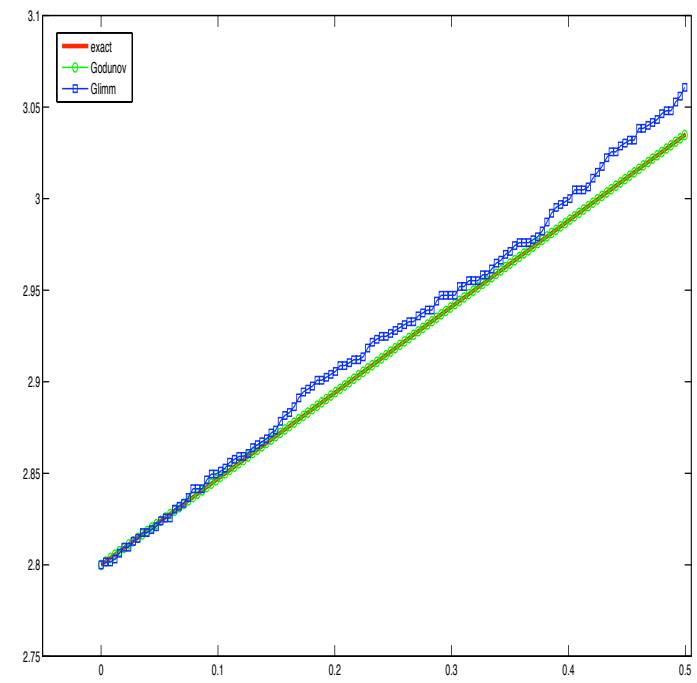

Fig. 6. Test case 5.1. Evolution of the 'mass': exact (continuous red line), Glimm (blue line with squares) and Godunov (green line with circles)

\subsubsection{Dam-break problem over a non-flat bottom topography}

The axis of the channel is the interval $[0,10]$ and the bottom topography is given by the function $H(x)=1-0.5 e^{-(x-5)^{2}}$. The initial condition is $q=0$ and

$$
h(x)= \begin{cases}H(x), & x \geq 4 ; \\ H(x)+0.5, & x<4 .\end{cases}
$$

The final time is $t=0.6$. Free boundary conditions are considered. The CFL parameter is set to 0.9 .

We consider a Roe scheme and a modified Lax-Friedrichs scheme which are consistent with the family of segments. Figure 7 shows the bottom topography and the free surface computed for both schemes using three meshes with increasing number of cells $(800,1600$ and 3200 cells respectively). Both schemes converge to the same solution. Moreover, the speed of propagation $\xi$ and the limit states $w^{-}$and $w^{+}$of the shock in the numerical solutions have been computed for both schemes by using a fine mesh of 32000 cells and the first divided difference as a smooth indicator. The value of the residual $\mid \xi\left(w^{+}-w^{-}\right)-$ $F\left(w^{+}\right)+F\left(w^{-}\right) \mid$obtained for the well-balanced Lax-Friedrichs scheme is 0.008 and for Roe scheme, 0.006 .

\subsubsection{Stationary contact discontinuities}

In this test we study the approximation of stationary contact discontinuities. Following the discussion in Section 4.2, such a discontinuity has to connect two states belonging 


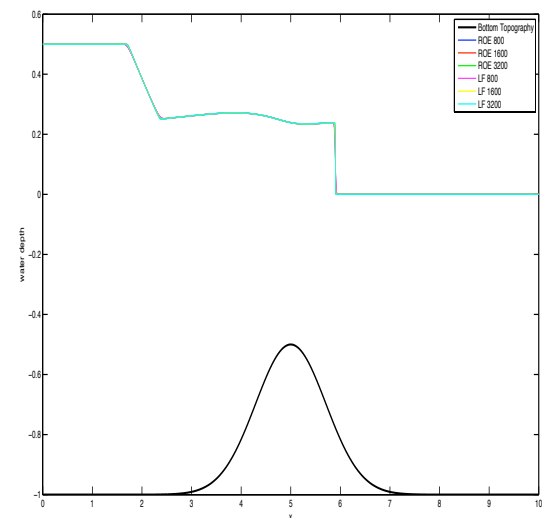

(a) Bottom topography and free surface at $\mathrm{t}=0.6$.

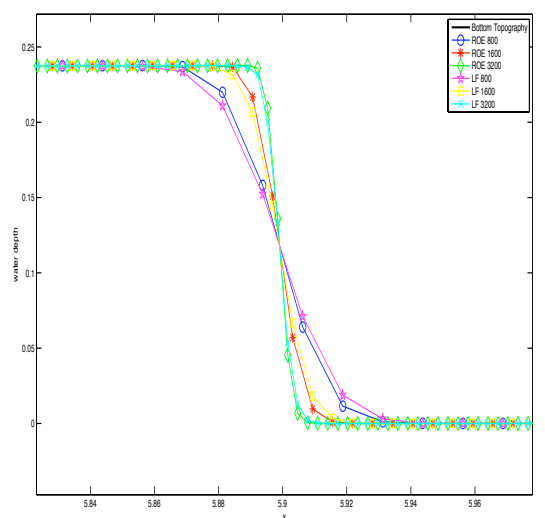

(b) Free surface (zoom) at $\mathrm{t}=0.6$.

Fig. 7. Test case 5.2.1. Dam-break problem: bottom topography and free surface at $t=0.6$.

to a same curve of the family (4.6). As the family of segments only satisfies (R1) for the particular case of the curves

$$
q=0, h-H=\text { const },
$$

the Roe and the modified Lax-Friedrichs schemes based on the family of segments are only expected to capture correctly stationary contact discontinuities corresponding to water at rest over a discontinuous bottom. To check this in practice, we consider a channel whose axis is the interval $[-5,5]$ and whose bottom is given by the function

$$
H(x)= \begin{cases}0, & x<0, \\ 1, & x>=0 .\end{cases}
$$

We consider the initial condition:

$$
w_{l}=\left[\begin{array}{c}
h_{l} \\
q
\end{array}\right]=\left[\begin{array}{c}
1 \\
\sqrt{4 g}
\end{array}\right], \quad w_{r}=\left[\begin{array}{c}
h_{r} \\
\sqrt{4 g}
\end{array}\right],
$$

where $h_{r}$ has been calculated so that both states belong to the same integral curve (4.6) $\left(h_{r} \cong 0.7892441190408083\right)$. The exact solution of this Riemann problem is thus a stationary contact discontinuity.

We have applied both schemes to this Riemann problem. As boundary condition, the state $w_{l}$ is imposed upstream and free boundary conditions downstream. The CLF parameter is set to 0.9 . Figures 8 and 9 show the stationary solutions obtained with both schemes using three meshes with increasing number of cells (100, 200 and 400 cells respectively). As expected, the numerical solutions do not converge to the exact solution. Note that both schemes converge to the same discontinuous function.

Let us check what happens if the numerical schemes are based on a family of paths satisfying (R1) for every integral curve of the linearly degenerate field. We consider the 

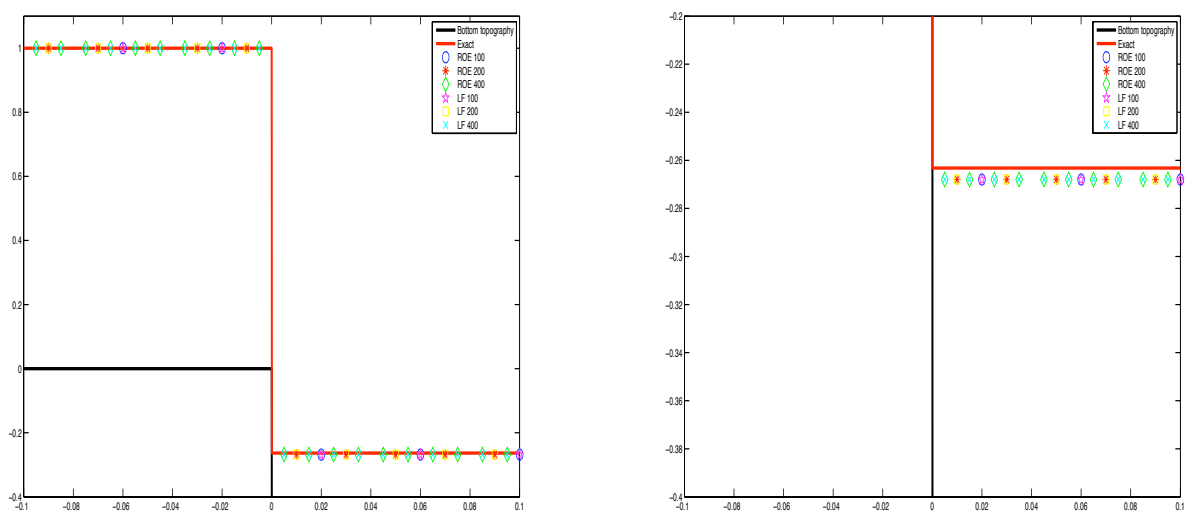

(a) Bottom topography and free surface (stationary (b) Bottom topography and free surface (stationsolution). ary solution): zoom

Fig. 8. Test case 5.2.2. Stationary contact discontinuity: Comparison between the modified Lax-Friedrichs and Roe schemes and the exact solution (free surface).

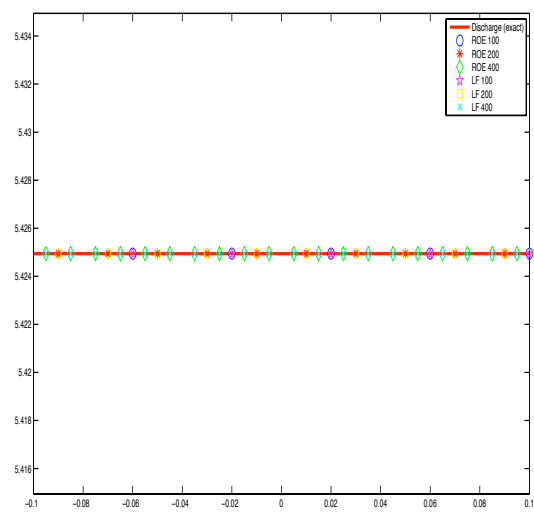

Fig. 9. Test case 5.2.2. Stationary contact discontinuity: Comparison between the well-balanced Lax-Friedrichs and Roe schemes and the exact solution (discharge).

family of paths constructed in [9] in order to design a Roe scheme which is well-balanced for every smooth stationary solution. The path connecting two states $W_{l}$ and $W_{r}$ consists of an arc of one of the curves of the family (4.6) and a segment lying on a plane $H=$ const: see [9] for details. Once the corresponding Roe matrix has been calculated, the construction of the modified Lax-Friedrichs scheme is straightforward.

Figure 10 shows the comparison between Roe, the modified Lax-Friedrichs scheme, and the exact solution for three meshes with increasing number of cells $(100,200$ and 400 cells respectively). As expected, the stationary contact discontinuity is exactly captured. 


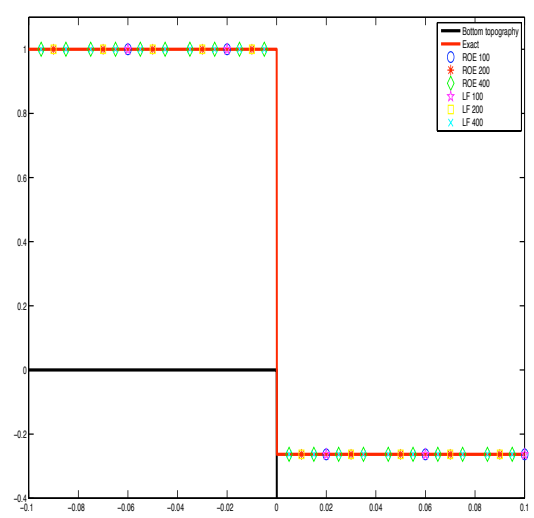

(a) Bottom topography and free surface (stationary (b) Bottom topography and free surface (station-
$\begin{array}{ll}\text { solution). } & \text { ary solution): zoom }\end{array}$

Fig. 10. Test case 5.2.2. Stationary contact discontinuity: Comparison between the exactly well-balanced Lax-Friedrichs and Roe schemes and the exact solution (free surface).

\subsection{Two-layer shallow water system}

\subsubsection{Approximation of internal shocks}

In this section we consider the discretization of the homogeneous two-layer shallow water system (that is, $H=$ cst.) by means of Roe and Lax-Friedrichs schemes. We check that the numerical solutions do not converge to the weak solutions involving shocks even when the same family of paths is used both for the definition of the jump conditions and the construction of the numerical scheme.

We begin with the family of segments for the definition of the jump condition (4.10). In this case:

$$
\begin{aligned}
& \int_{0}^{1} \Phi_{h_{1}}\left(s ; w_{l}, w_{r}\right) \frac{\partial \Phi_{h_{2}}}{\partial s}\left(s ; w_{l}, w_{r}\right) d s=\frac{h_{1}^{l}+h_{1}^{r}}{2}\left(h_{2}^{r}-h_{2}^{l}\right), \\
& \int_{0}^{1} \Phi_{h_{2}}\left(s ; w_{l}, w_{r}\right) \frac{\partial \Phi_{h_{1}}}{\partial s}\left(s ; w_{l}, w_{r}\right) d s=\frac{h_{2}^{l}+h_{2}^{r}}{2}\left(h_{1}^{r}-h_{1}^{l}\right) .
\end{aligned}
$$

In all of the cases considered here the order of the eigenvalues of the system is:

$$
\lambda_{\text {ext }}^{-}<\lambda_{\text {int }}^{-}<\lambda_{\text {int }}^{+}<\lambda_{\text {ext }}^{+} \text {. }
$$

Moreover

$$
\left|\lambda_{\text {int }}^{ \pm}\right|<<\left|\lambda_{\text {ext }}^{ \pm}\right| \text {. }
$$

We consider first a Lax-Friedrichs scheme consistent with the family of segments. Some easy calculations show that, for the particular choice of the family of segments, the last term in the modified equation (3.1) vanishes.

The goal is to compare the exact and the numerical Hugoniot curves corresponding to one of the internal characteristic fields; i.e. the fields related to the eigenvalues $\lambda_{\text {int }}^{ \pm}$). We proceed as follows: the state 


$$
w_{r}=\left[\begin{array}{c}
h_{1}^{r} \\
q_{1}^{r} \\
h_{2}^{r} \\
q_{2}^{r}
\end{array}\right]=\left[\begin{array}{c}
0.392034161025472 \\
-0.198826959396196 \\
1.588829011097482 \\
0.186046955388750
\end{array}\right]
$$

is fixed. Then, we compute the Hugoniot curve corresponding to the 'left 'states $w_{l}$ that can be connected with $w_{r}$ with a 3 -shock. To do this, we use the speed of the shock $\xi$ as a parameter and, for each value of $\xi$ we solve the non-linear system (4.10). In Figure 11 we show the projection (continuous blue line) of the computed Hugoniot curve onto the planes $h_{1}-q_{1}$ (left) and $h_{2}-q_{2}$ (right), respectively.

Next, we solve numerically a family of Riemann problems in which the right state is $w_{r}$, while $w_{l}$ runs on the Hugoniot curve. Using the first divided difference as a smooth indicator, the speed of propagation and the limit states of the shock corresponding to the eigenvalue $\lambda_{i n t}^{+}$is determined in the numerical solution. These calculations have been performed by using four meshes with decreasing step ( $\Delta x=0.002,0.001,0.0005$ and 0.00025). The numerical Hugoniot curves so obtained are compared with the exact one in Figure 11. Observe that the numerical solutions converge, but the limit is not a weak solution according to the chosen family of paths. Nevertheless, if $w_{r}$ and $w_{l}$ are close enough, both curves are very close. The same behaviour can be observed if the shock speed is close to zero: this situation corresponds in the figure to the intersections of the curves.
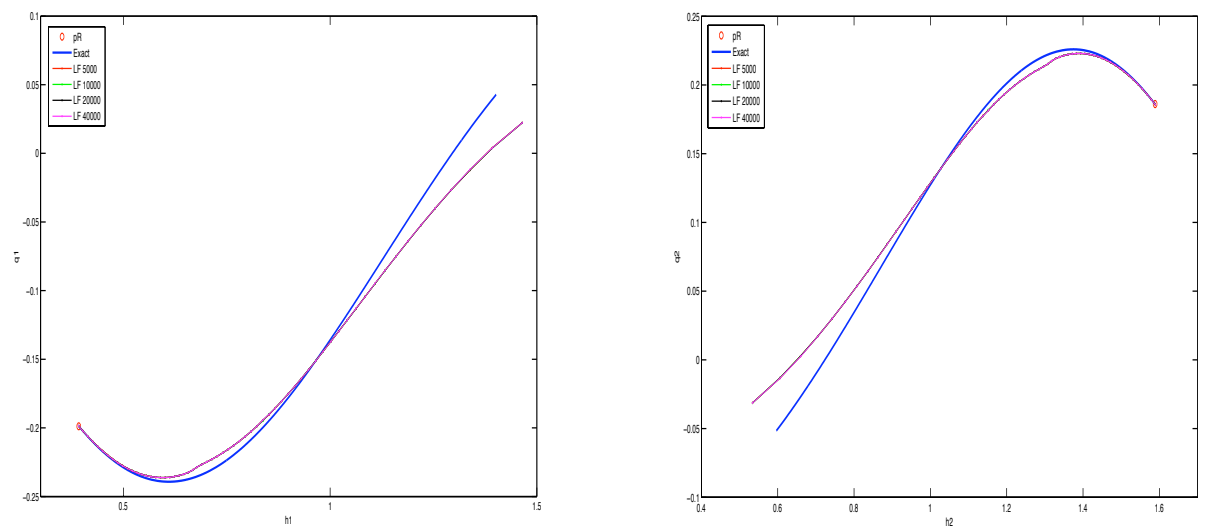

(a) Hugoniot curves (projection onto the plane $h_{1}-$ (b) Hugoniot curves (projection onto the plane $q_{1}$ ): exact (continuous blue line) and numerical $h_{2}-q_{2}$ ): exact (continuous blue line) and numerical (lines with dots) (lines with dots)

Fig. 11. Test case 5.3.1. Hugoniot curves: exact (continuous blue line) and numerical (lines with dots).

A similar behavior is observed for Roe scheme: the numerical approximations converge but the limit is not a weak solution according to the family of segments. Nevertheless, as the numerical viscosity of the scheme is smaller than that corresponding to Lax- 
Friedrichs, the results are expected to be closer to the exact solution: in Figure 12 we compare the exact Hugoniot curve with those computed with Lax-Friedrichs and Roe schemes using a mesh with 10000 cells.
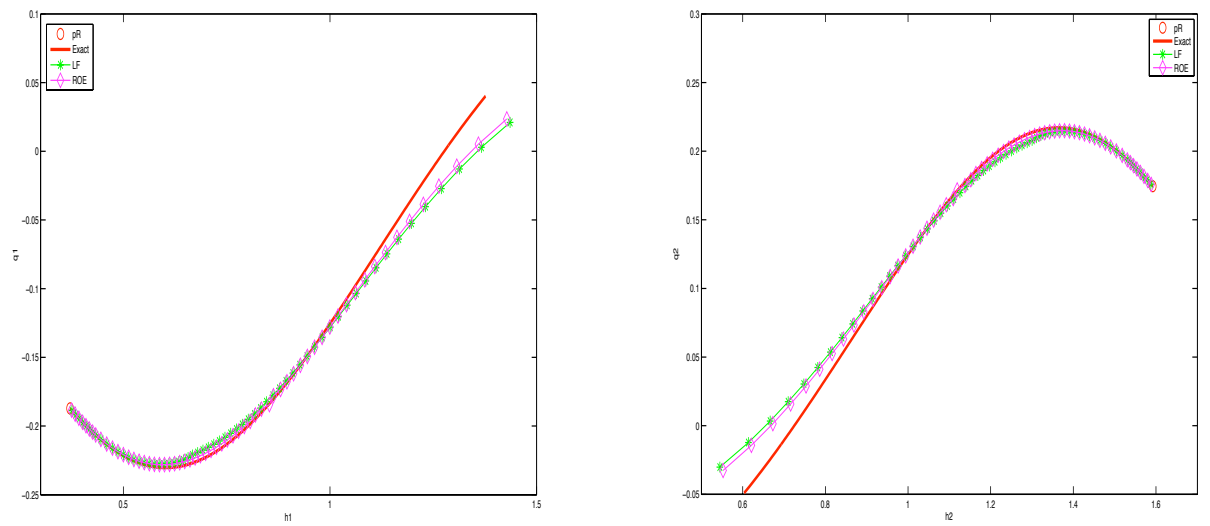

(a) Hugoniot curves (projection onto the plane $h_{1}-$ (b) Hugoniot curves (projection onto the plane $h_{2}-$ $q_{1}$ ): exact (continuous red line) and numerical (lines $q_{2}$ ): exact (continuous red line) and numerical (lines with dots) with dots)

Fig. 12. Test case 5.3.1. Hugoniot curves: exact (continuous red line) and numerical (lines with dots).

\subsubsection{Approximation of external shocks}

Due to the inequality (5.4)the CFL condition adjusts the numerical velocity to the external eigenvalues. As a consequence the effects of the numerical viscosity are much stronger for internal shocks and thus external shocks are expected to be better captured with Lax-Friedrichs or Roe schemes.

In order to check this, we proceed as in the previous test case: the state $w_{r}$ given by

$$
w_{r}=\left[\begin{array}{l}
0.257381469591567 \\
0.444901654188681 \\
0.110306344093418 \\
0.190672137450279
\end{array}\right]
$$

is fixed, and then the Hugoniot curves corresponding to the 'left 'states $\left(w_{l}\right)$ that can be connected with $w_{r}$ with a shock related to the eigenvalue $\lambda_{e x t}^{-}$are computed by solving the non-linear system (4.10) using $\xi$ as a parameter.

In Figure 13 we compare the exact Hugoniot curve with those computed with LaxFriedrichs and Roe scheme using a mesh with 2000 cells. Note how the curves are now much closer to each other than they were in the previous test case. 


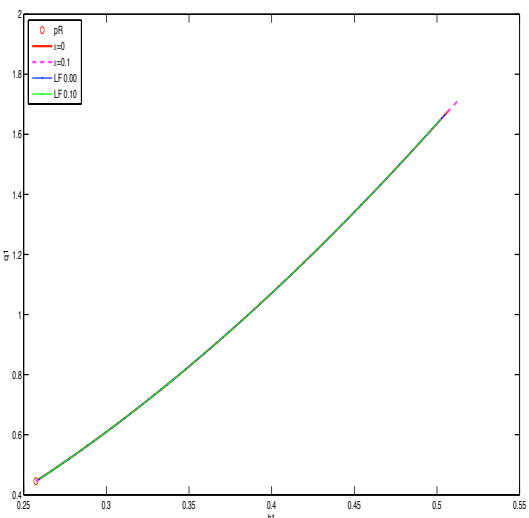

(a) Hugoniot curves (projection onto the plane $h_{1}-$ $q_{1}$ ): exact (continuous red line) and numerical (lines with dots)

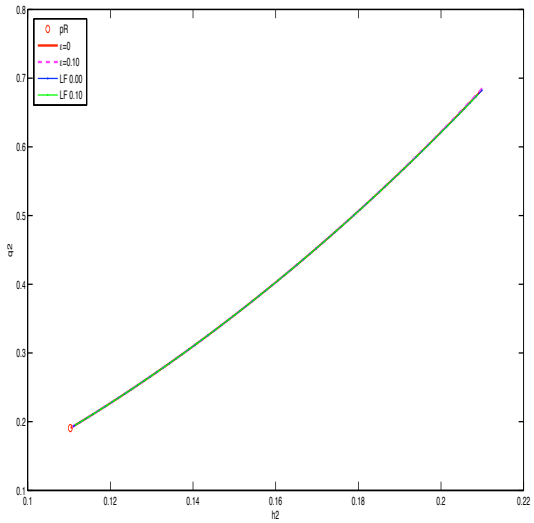

(c) Hugoniot curves (projection onto the plane $h_{2}-$ (d) Hugoniot curves (zoom of the projection onto $q_{2}$ ): exact (continuous red line) and numerical (lines the plane $h_{2}-q_{2}$ ): exact (continuous red line) and with dots) numerical (lines with dots)

Fig. 13. Test case 5.3.2. Hugoniot curves for Lax-Friedrichs and Roe schemes: exact (continuous red line) and numerical (lines with dots).

\subsection{Influence of the family of paths}

In this test we study the influence of small changes in the family of paths both in the weak and the numerical solutions. We consider now the jump conditions related to a different family of paths $\Phi_{h_{i}}^{\epsilon}\left(s ; w_{l}, w_{r}\right), i=1,2$. These curves are chosen so that:

$$
\begin{array}{ll}
h_{1}=\Phi_{h_{1}}^{\epsilon}\left(s ; w_{l}, w_{r}\right), & s \in[0,1] ; \\
h_{2}=\Phi_{h_{2}}^{\epsilon}\left(s ; w_{l}, w_{r}\right), & s \in[0,1] ;
\end{array}
$$


is a parameterization of the curve

$$
h_{2}=h_{2}^{l}+\left(\frac{h_{1}-h_{1}^{l}}{h_{1}^{r}-h_{1}^{l}}+\epsilon \frac{\left(h_{1}^{r}\right)^{2}-\left(h_{1}^{l}\right)^{2}}{\left(h_{1}^{r}\right)^{2}-\left(h_{1}^{l}\right)^{2}}\right) \frac{h_{2}^{r}-h_{2}^{l}}{1+\epsilon} .
$$

The jump conditions are now (4.10), with

$$
\begin{gathered}
\int_{0}^{1}\left(\Phi_{h_{1}} \frac{\partial \Phi_{h_{2}}}{\partial s}\right)\left(s ; w_{l}, w_{r}\right) d s=\frac{\left(h_{1}^{l}\right)^{2}(3+4 \epsilon)+2(3+2 \epsilon) h_{1}^{l} h_{1}^{r}+(3+4 \epsilon)\left(h_{1}^{r}\right)^{2}}{6(1+\epsilon)\left(h_{1}^{r}+h_{1}^{l}\right)}, \\
\int_{0}^{1}\left(\Phi_{h_{2}} \frac{\partial \Phi_{h_{1}}}{\partial s}\right)\left(s ; w_{l}, w_{r}\right) d s=\frac{h_{1}^{r}\left((3+4 \epsilon) h_{2}^{l}+(3+2 \epsilon) h_{2}^{r}\right)+h_{1}^{l}\left((3+2 \epsilon) h_{2}^{l}+(3+4 \epsilon) h_{2}^{l}\right)}{6(1+\epsilon)\left(h_{1}^{r}+h_{1}^{l}\right)} .
\end{gathered}
$$

Observe that the jump conditions of the previous tests are recovered for $\epsilon=0$.

As in Section 5.3.1, the state $w_{r}$ given by (5.5) is fixed and the exact Hugoniot curves are computed for different values of $\epsilon$. In Figure 14 the projections of the exact Hugoniot curves onto the planes $h_{1}-q_{1}$ (left) and $h_{2}-q_{2}$ (right) for the values $\epsilon=0.00,0.01,0.02$, 0.03, 0.04, 0.05, are shown.
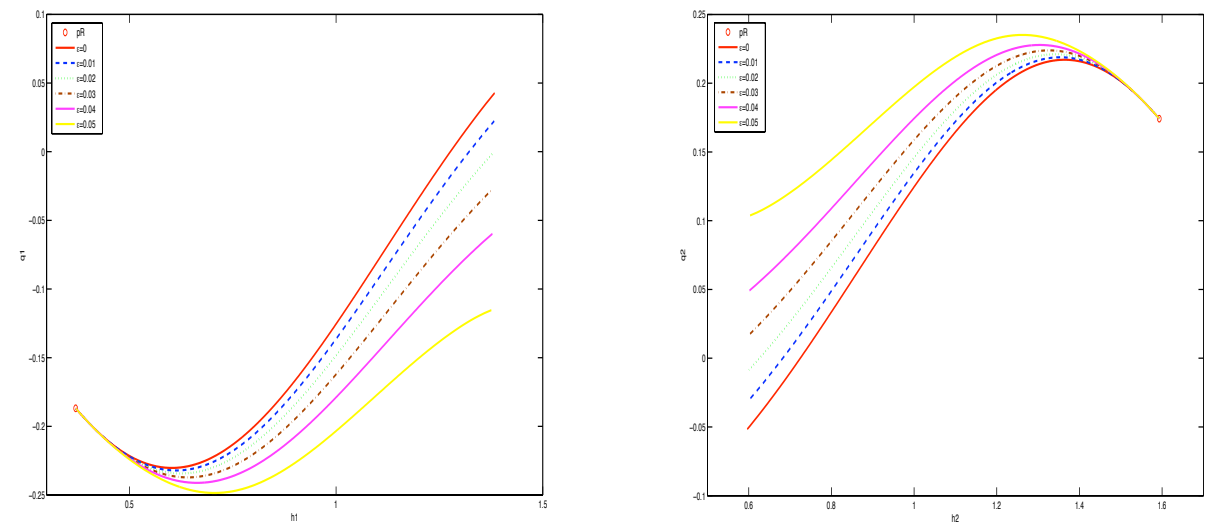

(a) Hugoniot curves (projection onto the plane $h_{1}-$ (b) Hugoniot curves (projection onto the plane $h_{2}-$ $\left.q_{1}\right)$ $\left.q_{2}\right)$

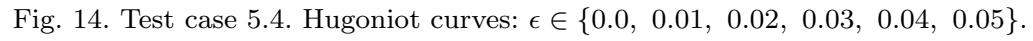

Next, we consider the Lax-Friedrichs schemes related to the new choice of the family of paths. An easy calculation shows again that, for this new family of paths, the last term of the modified equation (3.1) also vanishes. As a consequence, the second order modified equation is independent of $\epsilon$ : it coincides with the one corresponding to the family of segments.

We proceed as in the previous test case to compute the Hugoniot curves corresponding to the numerical scheme. In Figure 15 the curves obtained using a mesh with step $\Delta x=$ 0.001 for $\epsilon=0.00,0.01,0.02,0.03,0.04,0.05$, are depicted. Observe that all of the curves coincide: the Hugoniot curves obtained with the Lax-Friedrichs scheme for the different values of $\epsilon$ are reparameterizations of the same curve. This fact agrees with the fact that the second order modified equation is independent of $\epsilon$. 

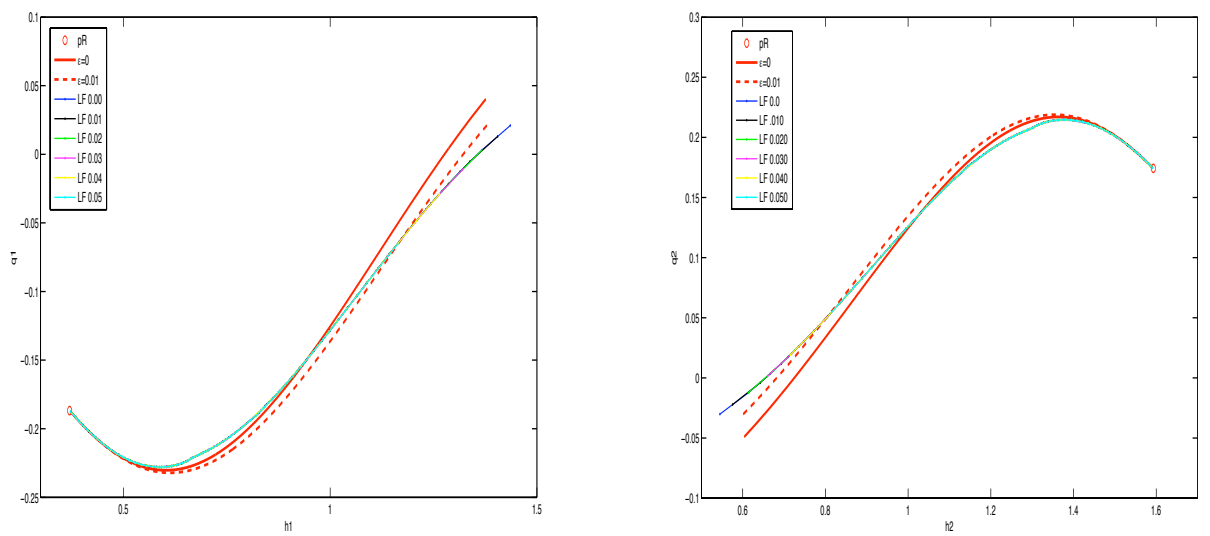

(a) Hugoniot curves (projection onto the plane $h_{1}-$ (b) Hugoniot curves (projection onto the plane $h_{2}-$ $\left.q_{1}\right)$ $\left.q_{2}\right)$

Fig. 15. Test case 5.4. Hugoniot curves for Lax-Friedrichs scheme with $\epsilon \in\{0.0,0.01,0.02,0.03,0.04,0.05\}$ : exact (red lines) and numerical (lines with dots).

Figure 16 shows the projections of the Hugoniot curve corresponding to Roe scheme for $\epsilon=0.00,0.01,0.02,0.03,0.04,0.05$ (line with dots) onto the planes $h_{1}-q_{1}$ and $h_{2}-q_{2}$. Note that, in this case, the obtained curves depend on $\epsilon$, but again the numerical solutions do not converge to the weak solutions corresponding to the chosen family of paths. Nevertheless, as in the previous test case, if $w_{r}$ and $w_{l}$ are close enough or the shock speed is close to zero, the exact and the numerical Hugoniot curves are also close. 


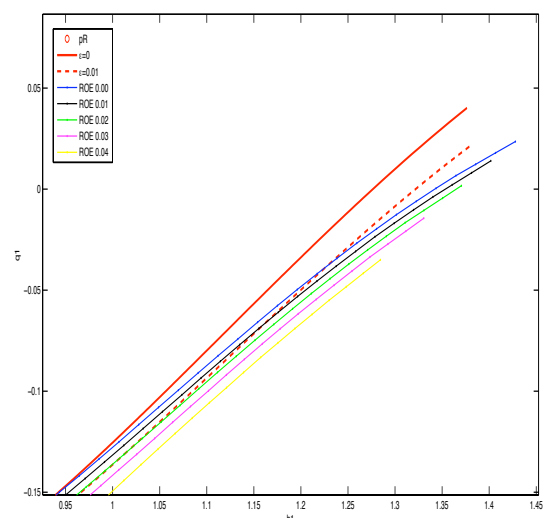

(a) 3-shock hugoniot curves (projection onto the (b) 3-shok hugoniot curves (projection onto the plane $\left.h_{1}-q_{1}\right)$

Fig. 16. Test case 5.4. 3-shock hugoniot curves (zoom) for Roe scheme with $\epsilon \in\{0.0,0.01,0.02,0.03,0.04,0.05\}$ : exact (red lines) and numerical (lines with dots).

\section{Conclusions}

When a hyperbolic system with nonconservative products and genuinely nonlinear fields is discretized, in order to be sure that the numerical approximations converge to a function which is a classical solution where it is smooth and whose discontinuities are in good agreement with the physics of the problem, the following steps should be taken $[19,16]$ :

- First, choose a regularization of the system which is consistent with the physics of the problem.

- Next, determine the DLM family of paths consistent with this regularization.

- Finally, design a numerical scheme whose solutions converge to weak solutions associated with this family of paths.

In practice, this strategy may be difficult to follow, since the actual calculation of a family of paths requires calculating regularized shock profiles associated with the given regularization. On the other hand, the convergence of the numerical solutions to the correct weak solutions is known for the Glimm scheme and the front tracking method [24], only; the implementation of these methods can be time consuming since they require the explicit knowledge of the corresponding Riemann solver.

In fact, when the nonconservative model under consideration is a simplified version of a more complex (but conservative) model - as is the case for the two-layer shallow water system - the above strategy may end up being more costly than than solving directly the more complex one. In these cases, the use of a numerical strategy based on a direct discretization of the nonconservative system by means of finite difference scheme which is formally path-consistent is advisable and may have the following advantages:

- The numerical solutions is formally consistent with the definition of the nonconservative product in the sense of Dal Maso, LeFloch, and Murat and, in turn, in the 
special case that the system admits a conservative subsystem, the numerical scheme is conservative for that subsystem in the sense of Lax.

- The approximations of the shocks provided by the schemes are consistent with a regularization of the system with higher-order terms that vanish as $\Delta x$ tends to 0 . (Obviously, the main drawback is that this regularization depends on the chosen family of paths and on the numerical scheme itself. This is issue dealt with in the present paper.)

- As originally pointed out by Hou and LeFloch [16] in the (simpler) case of scalar hyperbolic equations, the convergence error, measured in terms of our convergence error measure or in terms of the Hugoniot curves, is noticeable for very fine meshes, for discontinuities of great amplitude, and/or for large-time simulations, only.

- This strategy is extendable to high-order methods or to multidimensional problems, as developed, together with collaborators, by Coquel $[1,2,11]$ and Pares $[5,6]$.

The convergence error should also be compared with the experimental error. In the case of the two-layer shallow water system, the shocks captured by Roe scheme and the family of straightlines have been found [7] to be in good agreement with the experimental measurements of internal bores in the Strait of Gibraltar, despite of the simplicity of the family of paths.

In certain special situations, the convergence error measure is found to vanish identically. This is the case of systems whose nonconservative product is associated with a linearly degenerate field: for schemes that are formally consistent with a family of paths satisfying the condition (R1), then all of the discontinuities are correctly approximated and the scheme does converge to exact solutions. The discussion of linearly degenerate fields associated with nonconservative products was discussed earlier in $[19,26]$ from the theoretical standpoint and in [4] from the numerical standpoint. This problem may also exhibit an additional difficulty, the resonance problem, if one of the eigenvalues of the Jacobian matrix vanishes, and weak solutions may not be uniquely determined by their initial data, so that the limiting numerical solutions may depend both on the family of paths and the numerical scheme itself.

Acknowledgments

This research was partially supported by the Spanish Government Research project MTM2006-08075, by the A.N.R. (Agence Nationale de la Recherche) through the grant 06-2-134423, and by the Centre National de la Recherche Scientifique (CNRS). The authors are grateful to Frédéric Coquel for discussions on the matter of this paper.

References

[1] C. Berthon And F. Coquel, Nonlinear projection methods for multi-entropies Navier-Stokes systems, in "Innovative methods for numerical solutions of partial differential equations" (Arcachon, 1998), pp. 278-304, World Sci. Publ., River Edge, NJ, 2002.

[2] C. Berthon and F. Coquel, Nonlinear projection methods for multi-entropies Navier-Stokes systems, Math. of Comp. 76 (2007), 1163-1194. 
[3] C. Berthon, F. Coquel, and P.G. LeFloch, Why many theories of shock waves are necessary. Kinetic relations for nonconservative systems, in preparation.

[4] F. Bouchut, Nonlinear stability of finite volume methods for hyperbolic conservation laws and well-balanced schemes for sources, Frontiers in Math., Birkhuser Verlag, Bäsel, 2004.

[5] M.J. Castro, J.M. Gallardo, and C. Parés, High-order finite volume schemes based on reconstruction of states for solving hyperbolic systems with nonconservative products. Applications to shallow-water systems, Math. of Comp. 75 (2006), 1103-1134.

[6] M.J. Castro, J.M. Gallardo, and C. Parés, On a well-balanced high-order finite volume scheme for shallow water equations with topography and dry areas, J. Comput. Phys. 227 (2007), 574-601.

[7] M.J. Castro, J.A. García, J.M. GonzÁlez, J. Macías, C. Parés, and M.E. Vázquez, Numerical simulation of two layer shallow water flows through channels with irregular geometry, J. Comput. Phys. 195 (2004), 202-235.

[8] M.J. Castro, J. Macías, And C. PARÉs, A Q-Scheme for a class of systems of coupled conservation laws with source term. Application to a two-layer 1-D shallow water system, Math. Mod. Num. Anal. 35 (2001), 107-127.

[9] M.J. Castro, A. Pardo, and C. Parés, Well-balanced numerical schemes based on a generalized hydrostatic reconstruction technique, Math. Mod. Meth. App. Sci. 17 (2007), 2055-2113.

[10] M.J. Castro, A. Pardo, C. Parés, and E.F. Toro, Coefficient-splitting numerical schemes for nonconservative hyperbolic systems and high order extensions, in preparation.

[11] C. Chalons And F. Coquel, Numerical capture of shock solutions of nonconservative hyperbolic systems via kinetic functions. Analysis and simulation of fluid dynamics, Adv. Math. Fluid Mech., pp. 45-68, Birkhäuser, Bäsel, 2007.

[12] R. Courant and K.O. Friedrichs, Supersonic flow and shock waves, John Wiley, New York, 1948.

[13] G. Dal Maso, P.G. LeFloch, and F. Murat, Definition and weak stability of nonconservative products, J. Math. Pures Appl. 74 (1995), 483-548.

[14] B.T. Hayes and P.G. LeFloch, Nonclassical shocks and kinetic relations : scalar conservation laws, Arch. Rational Mech. Anal. 139 (1997), 1-56.

[15] B.T. Hayes and P.G. LeFloch, Nonclassical shocks and kinetic relations: finite difference schemes, SIAM J. Numer. Anal. 35 (1998), 2169-2194.

[16] T.Y. Hou And P.G. LeFloch, Why nonconservative schemes converge to wrong solutions: error analysis, Math. of Comput. 62 (1994), 497-530.

[17] P.D. Lax And B. Wendroff, Systems of conservation laws, Comm. Pure Appl. Math. 13 (1960), $217-237$.

[18] P.G. LeFloch, Entropy weak solutions to nonlinear hyperbolic systems in nonconservative form, Comm. Part. Diff. Equa. 13 (1988), 669-727.

[19] P.G. LeFloch, Shock waves for nonlinear hyperbolic systems in nonconservative form, Institute for Math. and its Appl., Minneapolis, Preprint \# 593, 1989.

[20] P.G. LeFlOCH, Entropy weak solutions to nonlinear hyperbolic systems in conservative form, Proc. Intern. Conf. on Hyperbolic problems, ed. J. Ballmann and R. Jeltsch, Note on Num. Fluid Mech., Vol. 24, Viewieg, Braunschweig, 1989, pp. 362-373.

[21] P.G. LeFloch, On some nonlinear hyperbolic problems (in English), Habilitation à Diriger des Recherches, Université de Paris 6, 1990.

[22] P.G. LeFloch, Hyperbolic systems of conservation laws: The theory of classical and nonclassical shock waves, Lectures in Mathematics, ETH Zürich, Birkhäuser, 2002.

[23] P.G. LeFloch, Graph solutions of nonlinear hyperbolic systems, J. Hyper. Diff. Equa. 1 (2004), 643-689.

[24] P.G. LeFloch And T.-P. Liu, Existence theory for nonlinear hyperbolic systems in nonconservative form, Forum Math. 5 (1993), 261-280. 
[25] P.G. LeFloch and M. Mohamadian, Why many shock wave theories are necessary. Fourth-order models, kinetic functions, and equivalent equations, J. Comput. Phys. 227 (2008), 4162-4189.

[26] P.G. LeFloch and M.D. Thanh, The Riemann problem for fluid flows in a nozzle with discontinuous cross-section, Comm. Math. Sci. 1 (2003), 763-796.

[27] P.G. LeFloch and M.D. Thanh, The Riemann problem for the shallow water equations with discontinuous topography, Comm. Math. Sc. 5 (2007), 865-885.

[28] M.L. Muñoz-Ruiz and C. PARÉs, Godunov method for nonconservative hyperbolic systems, Math. Method. Anal. Num. 41 (2007), 169-185.

[29] S. Noelle, Y. Xing, And C.W. Shu, High-order well-balanced finite volume WENO schemes for shallow water equation with moving water, J. Comput. Phys. 226 (2007), 29-58.

[30] C. PARÉs, Numerical methods for nonconservative hyperbolic systems: a theoretical framework, SIAM J. Numer. Anal. 44 (2006), 300-321.

[31] C. PARÉs and M.J. CAStro, On the well-balance property of Roe's method for nonconservative hyperbolic systems. Applications to shallow-water systems, Math. Model. Numer. Anal. 38 (2004), 821-852.

[32] J.B. SchiJf And J.C. Schonfeld, Theoretical considerations on the motion of salt and fresh water, in Proc. Minn. Int. Hydraulics Conv., joint meeting IAHR Hydro. Div. ASCE. (Sept. 1953) 1953, pp. 321-333.

[33] Y. XING AND C.-W. SHU, High order well-balanced finite volume WENO schemes and discontinuous Galerkin methods for a class of hyperbolic systems with source terms, J. Comput. Phys. 214 (2006), 567-598. 